OPEN ACCESS

Edited by:

Jane E. A. Reid,

Australian National University,

Australia

Reviewed by:

Fatah Kashanchi,

George Mason University,

United States

Elton J. R. Vasconcelos,

University of Leeds, United Kingdom

${ }^{*}$ Correspondence:

Damian F. J. Purcell

dfjp@unimelb.edu.au

${ }^{\dagger}$ Present address:

Georges Khoury and

Michelle Y. Lee,

Division of Microbiology and Immunology, Yerkes National

Primate Research Center, Emory University, Atlanta, GA, United States

Specialty section:

This article was submitted to

RNA

a section of the journal

Frontiers in Genetics

Received: 15 March 2021

Accepted: 17 May 2021

Published: 14 June 2021

Citation:

Khoury G, Lee MY

Ramarathinam SH, McMahon J, Purcell AW, Sonza S, Lewin SR and Purcell DFJ (2021) The RNA-Binding Proteins SRP14 and HMGB3 Control HIV-1 Tat mRNA Processing and Translation During HIV-1 Latency.

Front. Genet. 12:680725.

doi: 10.3389/fgene.2021.680725

\section{The RNA-Binding Proteins SRP14 and HMGB3 Control HIV-1 Tat mRNA Processing and Translation During HIV-1 Latency}

\author{
Georges Khoury't, Michelle Y. Lee ${ }^{1 t}$, Sri H. Ramarathinam², James McMahon', \\ Anthony W. Purcell ${ }^{2}$, Secondo Sonza ${ }^{1}$, Sharon R. Lewin ${ }^{3,4,5}$ and Damian F. J. Purcell ${ }^{1 *}$
}

${ }^{1}$ Department of Microbiology and Immunology, Peter Doherty Institute for Infection and Immunity, University of Melbourne, Melbourne, VIC, Australia, ${ }^{2}$ Infection and Immunity Program, Department of Biochemistry and Molecular Biology, Biomedicine Discovery Institute, Monash University, Clayton, VIC, Australia, ${ }^{3}$ Victorian Infectious Diseases Service, The Royal Melbourne Hospital at the Peter Doherty Institute for Infection and Immunity, Melbourne, VIC, Australia, ${ }^{4}$ Department of Infectious Diseases, The University of Melbourne at the Peter Doherty Institute for Infection and Immunity, Melbourne, VIC, Australia, ${ }^{5}$ Department of Infectious Diseases, Alfred Hospital and Monash University, Melbourne, VIC, Australia

HIV-1 Tat protein is essential for virus production. RNA-binding proteins that facilitate Tat production may be absent or downregulated in resting $\mathrm{CD} 4^{+} \mathrm{T}$-cells, the main reservoir of latent HIV in people with HIV (PWH) on antiretroviral therapy (ART). In this study, we examined the role of Tat RNA-binding proteins on the expression of Tat and control of latent and productive infection. Affinity purification coupled with mass spectrometry analysis was used to detect binding partners of MS2-tagged tat mRNA in a T cell-line model of HIV latency. The effect of knockdown and overexpression of the proteins of interest on Tat transactivation and translation was assessed by luciferase-based reporter assays and infections with a dual color HIV reporter virus. Out of the 243 interactions identified, knockdown of SRP14 (Signal Recognition Particle 14) negatively affected tat mRNA processing and translation as well as Tat-mediated transactivation, which led to an increase in latent infection. On the other hand, knockdown of HMGB3 (High Mobility Group Box 3) resulted in an increase in Tat transactivation and translation as well as an increase in productive infection. Footprinting experiments revealed that SRP14 and HMGB3 proteins bind to TIM-TAM, a conserved RNA sequence-structure in tat mRNA that functions as a Tat IRES modulator of tat mRNA. Overexpression of SRP14 in resting $C D 4^{+}$T-cells from patients on ART was sufficient to reverse HIV-1 latency and induce virus production. The role of SRP14 and HMGB3 proteins in controlling HIV Tat expression during latency will be further assessed as potential drug targets.

Keywords: HIV-1, latency, tat mRNA, SRP14, HMGB3, mRNA processing, translation

\section{INTRODUCTION}

The persistence of a reservoir of resting $\mathrm{CD} 4^{+} \mathrm{T}$-cells harboring silent provirus is the major impediment toward attaining a cure for HIV-1 (Deeks et al., 2016). Despite two decades of intensive investigation, the mechanisms contributing toward establishment and maintenance of latent infection in vivo remain incompletely understood (Khoury et al., 2018a). To date, blocks 
to the initiation of transcription have been the most widely studied and targeted for reversal of latent infection in clinical trials (Ait-Ammar et al., 2020). In these trials, reactivation of virus in the form of cell-associated HIV-1 RNA is detected but decay of the reservoir is almost never induced (Zerbato et al., 2019). Successful production of HIV-1 protein would be required to prime the immune response after reactivation of virus, and results of these trials suggest that additional blocks are present in the latently infected cell that prevent complete processing of RNA or translation to occur. The production of multiply spliced (MS) mRNA is required for successful production of HIV-1 virions, and these transcripts are not always detected after treatment with latency-reversing agents (Pasternak and Berkhout, 2018). We recently found that production of MS RNA is a better indication of virus reactivation ex vivo (Zerbato et al., 2021). In addition, a recent study showed that latent infection in blood $\mathrm{CD} 4^{+} \mathrm{T}$ cells from HIV-1 infected individuals on suppressive therapy was due to blocks in the post-initiation stages of transcription, including elongation, RNA-capping and splicing (Yukl et al., 2018).

The HIV-1 regulatory protein, Tat, is essential for successful transcription of the HIV-1 genome and virus production in natural infection (Ott et al., 2011). Whilst transcription initiation is a Tat-independent process, Tat is required for efficient elongation of transcription as the association of the negative factors DSIF (DRB Sensitivity-Inducing Factor) and NELF (Negative ELongation Factor) with the RNA polymerase II causes promoter-proximal pausing (Ping and Rana, 2001). Tat liberates its co-factor, the positive transcription elongation factor-b (P$\mathrm{TEFb}$ ), from its sequestration in the inactive 7SK snRNP complex (Krueger et al., 2010; Muniz et al., 2010), to associate with and phosphorylate DSIF and the carboxy-terminus of the stalled RNA polymerase II resulting in release of the transcriptional complex (Ping and Rana, 2001). Notably, Tat is also involved in the other post-transcriptional processes of RNA polyadenylation and splicing (Chiu et al., 2001, 2002; Schapira et al., 2003; Jablonski et al., 2010) where additional blocks to HIV-1 transcription in latent infection have been suggested. There are multiple pieces of evidence supporting the importance of Tat in diverting the integrated provirus away from latent infection. Fluctuations in the levels of Tat protein are a strong indicator of whether a cell will enter latency (Weinberger et al., 2005; Razooky et al., 2015). Nuclear retention of the multiply spliced RNAs that encode Tat or the presence of low levels of Tat protein contribute to maintaining the cells in a latent state (Lassen et al., 2006). Exogenous Tat delivered into latently infected cells inhibited proviral entry into latency, whilst established latency can be reversed by Tat (Donahue et al., 2012). Over time on suppressive therapy, mutations detrimental to Tat function accumulate, contributing to the persistence of latent provirus (Yukl et al., 2009).

We recently characterized the presence of an RNA regulatory element underlying Tat-encoding sequence, which we termed TIM-TAM (Tat IRES modulator of tat mRNA) (Khoury et al., 2020). We showed that TIM-TAM is involved in regulating latent infection, as viruses carrying a silent mutation disrupting the secondary structure of TIM-TAM resulted in a restriction in establishment of latency in primary $\mathrm{CD} 4^{+} \mathrm{T}$-cells. Furthermore, reactivation of latent HIV-1 from the CCL19 primary cell model (Saleh et al., 2011; Spina et al., 2013) infected with virus carrying mutated TIM-TAM was affected after treatment with PMA/PHA (Phorbol Myristate Acetate/PHytohAemagglutinin). The TIM-TAM was also shown to exhibit the properties of an internal ribosome entry site (IRES), RNA structures that facilitate translation initiation in a cap-independent manner.

Proteins required for processing and translation of tat mRNAs may be differentially expressed in cells carrying latent provirus and in cells undergoing productive infection. The recent study by Moron-Lopez et al. (2020) showed that human splice factors were differentially expressed between unstimulated and activated cells from ART-suppressed individuals. Activation of the splice acceptor 3 (SA3) site in the HIV-1 genome is required for production of tat mRNA, the use of which is tightly regulated by splicing silencers and enhancers (Saliou et al., 2009). In addition to translation initiation factors, auxiliary proteins unique to a particular IRES element may affect IRES function positively or negatively (Lozano and Martínez-Salas, 2015). Several proteins, including HNRNPA1, DDX3, hRIP and HuR (Monette et al., 2009; Rivas-Aravena et al., 2009; Liu et al., 2011) have been reported to impact the function of the HIV-1 5'UTR IRES element.

In this study, we used affinity purification coupled mass spectrometry analysis to identify cellular RNA-binding proteins that interact with tat mRNA during productive and latent infection. Using a principal component analysis-based scoring system, a short-list of thirteen proteins were chosen for followup investigation. Knockdown (KD) and overexpression assays were used to investigate the roles of these proteins in latent and productive infection of $\mathrm{HIV}-1$ with a dual-fluorescent reporter virus. The effect of $\mathrm{KD}$ of these proteins on the various stages of Tat expression-Tat mRNA splicing, Tat translation and Tat transactivation-were explored using luminescencebased reporter systems. The affinity purification approach and downstream validation assay systems allowed identification of Tat-RNA binding proteins that are differentially expressed in resting and activated $\mathrm{CD}^{+}{ }^{+}$T-cells isolated from people living with HIV on ART and are potential druggable targets for modulation of HIV-1 latency.

\section{MATERIALS AND METHODS}

\section{RNP Purification by MS2 Selection Affinity}

HIV 5'UTRtat1-Tat (NL4-3, nt 455-743; 5777-6044; 8369-8414) and Tat (nt 5,830-6,044; 8369-8414) fragments were amplified and cloned into NheI and EcoRI restriction sites of pcDNA3.1()::MS2 plasmid, upstream of $3 \times$ MS2 stem-loops (BamHIKpnI). RNAs were generated by run-off transcription with T7 MEGAscript kit (Promega) using KpnI linearized plasmids. DNA templates were digested with RQ1 RNase-Free DNase (Promega) and RNA were recovered by lithium chloride precipitation then dissolved in MilliQ water. J-Lat cells [clone 6.3, from NIH AIDS Reagent Program, (Jordan et al., 2003)] were expanded in RPMI supplemented with $10 \%$ FBS to $10^{8}$ cells per sample, left untreated or stimulated with TNF- $\alpha(20 \mathrm{ng} / \mathrm{mL})$ for $24 \mathrm{~h}$. Activation of virus 
expression was validated by flow cytometry ( $\mathrm{GFP}^{+}$expression) and p24 ${ }^{\text {CA }}$-ELISA as previously described (Khoury et al., 2020). Cells were then collected and lyzed with ice-cold lysis buffer (50 mM Tris-HCl pH 8, 1 mM EDTA, 150 mM NaCl, 0.1\% IgePal) supplemented with protease inhibitors (Roche) for $30 \mathrm{~min}$ at $4^{\circ} \mathrm{C}$. Protein cell extracts were dialyzed before use against buffer D $1 \times$ [Hepes KOH pH 7.920 mM, KCl 100 mM, glycerol 20\%, EDTA $0.2 \mathrm{mM}$, DTT $0.5 \mathrm{mM})+\mathrm{MgCl} 23 \mathrm{mM}+$ protease inhibitor tablet (Roche)] for $2 \mathrm{~h}$ at $4^{\circ} \mathrm{C}$, followed by centrifugation for $10 \mathrm{~min}$ at $1,700 \times \mathrm{g}$ at $4^{\circ} \mathrm{C}$. Five hundred pmol of MS2-tagged RNAs into $100 \mu \mathrm{l}$ buffer $\mathrm{D} 1 \times$ were denatured by $10 \mathrm{~min}$ heating at $65^{\circ} \mathrm{C}$, followed by slow cooling at room temperature with addition of $7.75 \mu \mathrm{l}$ of $62.5 \mathrm{mM} \mathrm{MgCl}_{2}$ to a final concentration of $4.5 \mathrm{mM} \mathrm{MgCl}_{2}$. After $10 \mathrm{~min}$ incubation at room temperature, RNAs were incubated with a fivefold molar excess of purified MBP-MS2 fusion protein at $4^{\circ} \mathrm{C}$ for $20 \mathrm{~min}$. The RNA-MS2:MS2MBP complexes formed were incubated with amylose beads (200 $\mu \mathrm{l}$, GE Healthcare) equilibrated in buffer $\mathrm{D}$ for $2 \mathrm{~h}$ at $4^{\circ} \mathrm{C}$. After three washes with $500 \mu \mathrm{l}$ of buffer D, $1 \mathrm{mg}$ of protein extract supplemented with $5 \mu \mathrm{M}$ of yeast tRNAs (Sigma-Aldrich) was added. After 20 min of incubation at $4^{\circ} \mathrm{C}$ with constant agitation, three successive washes were performed in Buffer D and RNP complexes eluted twice by incubation for $20 \mathrm{~min}$ at room temperature with $200 \mu \mathrm{l}$ of Buffer D containing $10 \mathrm{mM}$ maltose. Half of the eluted RNP complexes were processed in solution for mass spectrometry analyses. For western-blot analysis, $10 \%$ of the eluted material was used. Experiments were repeated three independent times using different batches of RNA and protein lysate.

\section{Mass Spectrometry Analysis}

TCEP (10 mM, Thermo Fisher Scientific) was added to the eluted sample to reduce the cysteine bonds in proteins, and heated for $20 \mathrm{~min}$ at $60^{\circ} \mathrm{C}$ followed by alkylation of cysteines using $25 \mathrm{mM}$ Iodoacetamide (Sigma-Aldrich) for $20 \mathrm{~min}$ at RT in the dark. Samples were subjected to proteolytic cleavage by addition of $1 \mu \mathrm{g}$ of trypsin protease from porcine pancreas per $100 \mu \mathrm{g}$ of protein and incubated overnight at $37^{\circ} \mathrm{C}$ under agitation. Reactions were stopped by addition of formic acid and the resultant peptides were concentrated using C18-packed tips (BondElut, Agilent/Varian). The peptides were subject to online trapping using a PepMap100 trap column at $15 \mu \mathrm{L} / \mathrm{min}$ followed by separation on PepMap $100 \mathrm{C} 18$ nanocolumn $(50 \mathrm{~cm} \times 75 \mu \mathrm{m})$ using a 30 -min gradient of Buffer B ( $80 \%$ ACN $0.1 \%$ Formic acid) over Buffer A (0.1\% Formic acid). The online separated peptides were analyzed using a Q-Exactive plus mass spectrometer (Thermo Fisher Scientific, Bremen, Germany). The survey scans were acquired at 70,000 resolution from 375 to $1,800 \mathrm{~m} / \mathrm{z}$, the ion accumulation target was set to $3 \mathrm{e} 6$ with maximum injection time of $120 \mathrm{~ms}$. A total of 12 most intense ions (with charge more than 2) were sequentially isolated and fragmented by higherenergy collisional dissociation (HCD) set to $27 \%$, at a resolution of 17,500, target of $1 \mathrm{e} 5$ ions and maximum injection time of $120 \mathrm{~ms}$. To identify protein groups, data acquired was converted into mgf and searched against Swissprot human database (version 2016_12) using ProteinPilot software (v4.0, SCIEX) with the following search parameters: Iodoacetamide alkylation, trypsin enzyme digestion, instrument-specific settings for TripleTOF $5600+($ MS tolerance $0.05 \mathrm{Da}, \mathrm{MS} / \mathrm{MS}$ tolerance $0.1 \mathrm{Da}$, charge state $+2-+5$ ), biological modification probabilistic features on, thorough ID algorithm, and detected protein threshold 0.05. Mass Spectrometry Interaction Statistics (MiST) (Jäger et al., 2011) analysis was conducted to sort proteins based on specificity, reproducibility and abundance over the three replicates. The mass spectrometry proteomics data have been deposited to the ProteomeXchange Consortium via the PRIDE (Perez-Riverol et al., 2019) partner repository with the dataset identifier PXD025782.

\section{Generation of Knockdown RFP ${ }^{+}$Jurkat Cell Lines}

Three or four Sherwood UltramiR shRNA viral particles $\left(10^{6}-10^{7} \mathrm{TU} / \mathrm{mL}\right)$ against each of the 15 protein targets of interest were obtained from TransOmic Technologies. The pZIP (SFFV) shRNA-mir lentivectors constitutively express the short hairpin RNA (shRNA-mir), puromycin selection marker and red fluorescent protein (RFP) driven by the Spleen Focus Forming Virus (SFFV) promoter. Jurkat cells $\left(2.1 \times 10^{5}\right)$ were transduced with $100 \mu \mathrm{L}$ of pooled pseudoviruses for each protein target separately at a MOI of 2.5. Spinoculation was conducted at 1,200 $\times \mathrm{g}$ for $2 \mathrm{~h}$ at $23^{\circ} \mathrm{C}$ in a flat-bottom 96-well plate. Cells were incubated at $37^{\circ} \mathrm{C}$ for $72 \mathrm{~h}$ then sorted on $\mathrm{RFP}^{+}$expression on an Astrios cell sorter (Beckman Coulter). Bulk populations were then kept in culture under $0.7 \mu \mathrm{g} / \mathrm{mL}$ puromycin selection. Single clones expressing high levels of $\mathrm{RFP}^{+}$cells were selected from the bulk population on the BD FACSAria III. Bulk and single clones were maintained under constant puromycin selection then surviving clones were expanded. Cells were collected for RT-qPCR analysis as well as immunoblotting.

\section{Cloning of Tat Expression Reporter Constructs}

For the Tat/GH1 constructs, HIV-1 sequences were derived from pNL4-3 (M. Martin, NIH, Bethesda, MD, United States), GH1 sequences were amplified from pØhGH (Nichols Institute Diagnostics), LucF from pGL4.13[luc2/SV40] (Promega), and LucR from pGL4.73[hRluc/SV40] (Promega). These segments were assembled by SOE-PCR and cloned into the pcDNA3.1(Invitrogen) backbone via use of the Xhol and XbaI sites. The NT5C3 splicing constructs were cloned into the pcDNA3.1backbone through cleavage of NhoI and NheI where the NT5C3 cellular sequences were amplified from HeLa cell genomic DNA and the other components derived as for the Tat/GH1 constructs.

\section{DNA Transfection of KD RFP + Cells and Luciferase Analysis}

Bulk populations or single clones of the $\mathrm{KD} \mathrm{RFP}^{+}$Jurkat cell lines $\left(6 \times 10^{4}\right.$ cells) were plated into a round-bottom 96-well plate. Media was completely removed and cells were transfected with $1.6 \mu \mathrm{g}$ reporter constructs using DMRIE-C reagent (1:2.5, Invitrogen) in $62.5 \mu \mathrm{L}$ Opti-MEM (Gibco). For transactivation assays, $200 \mathrm{ng}$ of Tat WT and $1 \mu \mathrm{g}$ of Tat hGH reporter construct with $300 \mathrm{ng}$ of both LTR-lucFirefly and lucRenilla. For translation 
assays, $300 \mathrm{ng}$ of Tat-Cap or IRES LucF reporter constructs were used with $300 \mathrm{ng}$ of lucRenilla for normalization. For splicing assays, $300 \mathrm{ng}$ of $\mathrm{Tat}^{+}$or Tat- reporter constructs with $300 \mathrm{ng}$ of LTR-lucFirefly. Three hours post-transfection, DNA:DMRIE$\mathrm{C}$ mix was removed and media replenished. Twenty-four hours post-transfection, cells were collected and luciferase activity was measured following lysis in $40 \mu \mathrm{L}$ of $1 \mathrm{X}$ passive lysis buffer using a FLUOstar plate reader with the dual-luciferase reporter assay (Promega).

\section{Virus Production and Transduction}

R7GEmTB dual color reporter virus was produced by replacing mCherry into R7GEmC [obtained from NIH AIDS Reagent Program, (Calvanese et al., 2013)] with mtagBFP2 fluorescent protein. SRP14 and PTB cDNA were cloned into pInducer10 lentivector (Meerbrey et al., 2011) by replacing tRFP-shRNA cassette with protein ORF-T2A-mtagBFP2. Viral stocks were generated by transfecting the proviral constructs into HEK 293T cells with Lipofectamine 2000 (Invitrogen) in serum free media (Opti-MEM, Gibco). Supernatants were collected after $72 \mathrm{~h}$, clarified by centrifugation and $0.45 \mu \mathrm{m}$ filtration to clear cell debris. Particles were concentrated using microcon centrifugal filter device (30K, Merck Millipore) or pelleted by overlaying supernatant on a cushion of $20 \%(\mathrm{w} / \mathrm{v})$ sucrose in TNE buffer (10 mM Tris- $\mathrm{HCl} \mathrm{pH} 8,1 \mathrm{mM}$ EDTA, $150 \mathrm{mM} \mathrm{NaCl}$ ) in Ultra Clear Thinwall tubes and centrifugation at 24,200 rpm for $2 \mathrm{~h}$ at $4^{\circ} \mathrm{C}$ (Beckman SW28/SW41Ti rotor). Viral particle pellets were resuspended into Opti-MEM and stored at $-80^{\circ} \mathrm{C}$. Virus titres were quantified by measuring $\mathrm{p} 24^{\mathrm{CA}}$ levels by capture ELISA as previously described (Khoury et al., 2020) and titration into TZM-bl cells (Sarzotti-Kelsoe et al., 2014). KD RFP ${ }^{+}$Jurkat cells and $\mathrm{CD}^{+}$T-cells $\left(10^{6}\right.$ cells) were infected with 40 and 100 ng R7GEmTB virus (+Env 92HT593.1), respectively. Cells were spinoculated at $1,200 \times \mathrm{g}$ for $2 \mathrm{~h}$ at $23^{\circ} \mathrm{C}$ in a 96 well flat-bottom plate and incubated at $37^{\circ} \mathrm{C}$ for 3 days. Cells were collected, washed and resuspended in $100 \mu \mathrm{L}$ of $1 \mathrm{X}$ PBS then loaded onto cover slips that had been pre-coated with poly-LLysine $0.01 \%$ (Sigma-Aldrich). Cells were incubated for $1 \mathrm{~h}$ on the coverslip then rinsed with PBS 1X and fixed with 2\% PFA. Cells were treated with glycine $0.2 \mathrm{M}$ for $10 \mathrm{~min}$ at room temperature then rinsed with PBS $1 \mathrm{X}$ and $\mathrm{H}_{2} \mathrm{O}$. Coverslips were inverted on a microscope slide containing ProLong Gold Antifade Mountant (Thermo Fisher Scientific). Images were acquired on LSM710 confocal microscope using Zeiss Zen software. J-Lat 10.6, 8.4 and A2 (obtained from the NIH AIDS reagent program) were infected via spinoculation at $1,500 \times \mathrm{g}$ for $2 \mathrm{~h}$ at $23^{\circ} \mathrm{C}$ followed by $1 \mathrm{~h}$ incubation at $37^{\circ} \mathrm{C}$ before replenishing the culture with fresh media without or with $5 \mu \mathrm{g} / \mathrm{mL}$ doxycycline. Two days postinfection, cells were washed and stained with Near-IR Live/Dead fixable dead cell staining (Invitrogen). Finally, cells were washed, fixed with $1 \%$ formaldehyde and acquired using a Fortessa flow cytometry instrument (BD Bioscience). Analysis was performed using FlowJo Software, version 10.4.2.

\section{Ethics}

The studies involving the use of blood samples from HIV negative donors were reviewed and approved by the Human Research and Ethics Committees from the University of Melbourne (1509VIC-03 and 17-08VIC-01). All HIV-1 seronegative donors were recruited by the Red Cross Blood Bank (Melbourne, Australia) and provided written informed consent for the use of their blood products for the research. The use of blood samples from people living with HIV was approved by the Alfred Hospital (HREC214/15) for the study entitled Large volume peripheral blood mononuclear cells (PBMCs) collection by leukapheresis to define HIV persistence in HIV-infected adults. All participants provided informed consent and the protocol was approved by the local Institutional Review Board.

\section{Participant Details}

PBMCs from people living with HIV on ART with a viral load $<20$ copies/mL for $\geq 3$ years were collected by leukapheresis (Alfred Hospital, Melbourne, Australia; Supplementary Table 1) and stored in liquid nitrogen. Resting CD4 ${ }^{+}$T-cells (purity $>95 \%$ ) were isolated from PBMCs by negative selection using $\mathrm{CD}^{+}{ }^{+}$T-cell isolation kit (Miltenyi Biotec) supplemented with anti-CD69 (clone L78, BD) and anti-HLA-DR (clone 2-O6). $\mathrm{CD}^{+}{ }^{+}$T-cells were activated with anti-CD3/CD28 (coated $\alpha$-CD3 clone OKT3, BD, $1 \mu \mathrm{g} / \mathrm{mL}$ and soluble $\alpha$-CD28 clone $\mathrm{L} 293, \mathrm{BD}, 0.5 \mu \mathrm{g} / \mathrm{mL}$ ) for $48 \mathrm{~h}$ at $37^{\circ} \mathrm{C}$.

\section{Western-Blot}

Resting and activated $\mathrm{CD}^{+}{ }^{+}$T-cells isolated from patients under ART $\left(30 \times 10^{6}\right.$ cells) were lysed for $30 \mathrm{~min}$ on ice using ice-cold IGEPAL cell lysis buffer (Tris- $\mathrm{HCl}$ pH $8.850 \mathrm{mM}$, $\mathrm{NaCl} 150 \mathrm{mM}$, IGEPAL 1\%, EDTA $1 \mathrm{mM}$ ) supplemented with protease inhibitor cocktail (Complete ${ }^{\mathrm{TM}}$, Mini, EDTA-free Protease Inhibitor Cocktail, Roche). Cell lysate was cleared and quantified using the Bio-Rad Protein Assay Reagent (Bio-Rad). Equal amounts of each sample $(20 \mu \mathrm{g})$ were loaded on $15 \%$ SDS-PAGE, transferred to nitrocellulose membrane. Blots were probed with anti-SRP14 (B-3, sc-377012, Santa Cruz) at 1:10, anti-HMGB3 (clone 546519, R\&D) 1:1,000, anti-PTB (clone 7, \#325000, Invitrogen) at 1:500 and anti-GAPDH (14C10, Cell Signaling) at 1:1,000. Antibodies were detected using either goat anti-rabbit (Invitrogen, \#656120) or goat anti-mouse $\operatorname{IgG}(\mathrm{H}+\mathrm{L})$ HRP (Invitrogen, \#626520) at 1/5,000 and developed with SuperSignal West Pico Chemiluminescent Substrate (Thermo Fisher Scientifc). Images were visualized using an MF-ChemiBis 3.2 imaging system (DNR).

\section{Quantitative Real-Time PCR Analysis}

RNA from resting and activated $\mathrm{CD} 4^{+}$T-cells isolated from patients under ART $\left(15 \times 10^{6}\right.$ cells $)$ and bulk and individual clone of interest for the KD RFP + Jurkat cells as well as untransduced Jurkats were extracted using TRIzol following the manufacturer's protocol. RNA (500 ng) was DNase-treated with RQ1 DNase (Promega) and reverse transcribed using $4 \mathrm{U}$ OmniScript (Qiagen) with dNTP $(0.5 \mathrm{mM})$, random hexamers $(2 \mu \mathrm{M})$, oligo $(\mathrm{dT})_{15}(1 \mu \mathrm{M})$, and RNasin $(10 \mathrm{U})$ in $30 \mu \mathrm{L}$ reactions for $1 \mathrm{~h}$ at $37^{\circ} \mathrm{C}$. Real-time PCR was performed on CFX Connect real-time PCR detection system (Bio-Rad) using Fast SYBR Green Master Mix (Applied Biosystems), forward/reverse primers (Supplementary Table 2) at $500 \mathrm{nM}$ final concentration, 
$2 \mu \mathrm{L}$ of undiluted cDNA in reactions with a final volume of $20 \mu \mathrm{L}$. Each sample was assayed in duplicate and normalized on GAPDH content and untransduced Jurkat cells for KD RFP + Jurkats or $18 \mathrm{~S}$ content and activated CD4 ${ }^{+}$T-cells for primary $\mathrm{CD}^{+}{ }^{+} \mathrm{T}$-cells. Cycling conditions used were $95^{\circ} \mathrm{C}$ for $10 \mathrm{~min}$ for activation of the DNA polymerase followed by 45 cycles of denaturation at $95^{\circ} \mathrm{C}$ for $3 \mathrm{~s}$ and annealing/extension at $60^{\circ} \mathrm{C}$ for $20 \mathrm{~s}$. Melt curve analysis was performed each time with a starting temperature of $60^{\circ} \mathrm{C}$, increasing to $95^{\circ} \mathrm{C}$ with $0.5^{\circ} \mathrm{C}$ increments every $0.05 \mathrm{~s}$. Data were analyzed on CFX Manager 3.0 software employing $2^{-\Delta \Delta \mathrm{Ct}}$ to determine fold changes.

\section{T-Cell Electroporation}

Electroporation of resting $\mathrm{CD} 4^{+}$T-cells was performed using an Amaxa human T-cell Nucleofector kit (Lonza). Purified resting $\mathrm{CD}^{+}{ }^{+}$T-cells $\left(5.3 \times 10^{6}\right)$ were re-suspended in $100 \mu \mathrm{l}$ nucleofector solution (4.5:1 ratio of human T-cell nucleofector solution:supplement) and transfected with $1.2 \mu \mathrm{g}$ DNA per $10^{6}$ cell using Amaxa nucleofector program U-014 for high viability. Cells were then transferred into a 12 -well plate containing preequilibrated media, followed by half-media change $6 \mathrm{~h}$ postnucleofection. Cells were maintained for $48 \mathrm{~h}$ at $37^{\circ} \mathrm{C}$ in media supplemented with $1 \%$ FBS and $1 \mathrm{U} / \mathrm{mL}$ IL-2, with or without $5 \mu \mathrm{g} / \mathrm{mL}$ doxycycline. After 2 days, cells were collected and stained for CD25 (PE-Cy7, clone 2A3, BD) and HLA-DR (V605, clone L243 BioLegend) activation markers in comparison to PHA stimulated $(10 \mu \mathrm{g} / \mathrm{mL})$ cells. Culture supernatant was also harvested and viral RNA was isolated using QIAamp Viral RNA Mini Kit (Qiagen) following the manufacturer's protocol.

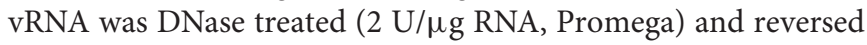
transcribed using Omniscript as described above. HIV RNA levels were assessed using droplet digital PCR (ddPCR) using pol primers/probe (Supplementary Table 2). Thermal cycling was conducted as follows: $95^{\circ} \mathrm{C}$ for $10 \mathrm{~min}, 40$ cycles of $94^{\circ} \mathrm{C}$ for $30 \mathrm{~s}$ and $60^{\circ} \mathrm{C}$ for $60 \mathrm{~s}$, followed by $98^{\circ} \mathrm{C}$ for $10 \mathrm{~min}$ (ramp rate $2^{\circ} \mathrm{C} / \mathrm{s}$ for each step) on a C1000 Touch Thermal cycler (Bio-Rad). The droplets were subsequently read on a QX200 droplet reader (BioRad) and the data were analyzed with Quanta-Soft 1.7.4 software. The limit of detection of our assay was of 58 copies $/ \mathrm{mL}$.

\section{Recombinant Proteins}

Production and purification of MBP-MS2 protein was done as previously described (Deckert et al., 2006). cDNA encoding SRP14, HMGB3 and PTB were cloned into NheI and HindIII restriction sites of pET28a + bacterial vector (Novagen) allowing the expression of $\mathrm{N}$-terminally $6 \times$-His tagged proteins. All recombinant proteins were expressed in E. coli BL21 (DE3) Codon + bacteria grown in LB/Kanamycin and induced by addition of $0.1 \mathrm{mM}$ IPTG overnight at $37^{\circ} \mathrm{C}$ (for $\mathrm{PTB}$ and SRP14) or $30^{\circ} \mathrm{C}$ (for HMGB3). Cells were sonicated in lysis buffer containing $20 \mathrm{mM}$ potassium phosphate $\mathrm{pH} 7.4$ ( $\mathrm{pH}$ to 6 for HMGB3), $1 \mathrm{M} \mathrm{KCl}, 1 \mathrm{mM}$ DTT, $20 \mathrm{mM}$ imidazole and protease inhibitor cocktail (Roche), then treated with $1.5 \mathrm{mg} / \mathrm{mL}$ lysozyme (from chicken egg white, Sigma-Aldrich) for $20 \mathrm{~min}$ on ice. Soluble proteins were purified by polyethyleneimine $(0.4 \%$ final $)$ followed by ammonium sulfate precipitation (30\% for SRP14 and PTB; $40 \%$ then $60 \%$ precipitation for HMGB3). Proteins were purified by affinity purification using Ni-NTA affinity resin (Qiagen) coupled to an Äkta pure FPLC (GE Healthcare). The bound proteins were eluted with a linear imidazole gradient (20 $\mathrm{mM}$ to $500 \mathrm{mM}$ ). Proteins were buffer exchanged into buffer D (20 mM HEPES-KOH pH 7.9, $100 \mathrm{mM} \mathrm{KCl,} 0.2 \mathrm{mM}$ EDTA, $3 \mathrm{mM} \mathrm{MgCl}_{2}, 0.5 \mathrm{mM}$ DTT, 20\% glycerol) using PD10 desalting columns (GE Healthcare) followed by concentration using 3, 10, and $30 \mathrm{k}$ cutoff amicon columns (Merck) for SRP14, HMGB3 and PTB, respectively. Proteins concentration was determined using UV spectroscopy at $280 \mathrm{~nm}$. Purity was confirmed by $12.5 \%$ SDS-PAGE and western-blot analysis using protein specific antibodies and anti-His HRP conjugated antibody (clone J099B12, BioLegend) at 1:500.

\section{Footprinting Assays}

Footprinting analysis was performed by SHAPE as previously described (Mortimer and Weeks, 2007). Briefly, tat2 RNA was generated by run-off transcription using Sp6 MEGAscript kit (Promega) and pSP65::tat 2 construct linearized with ClaI. In vitro transcribed tat 2 RNA (1 pmol) was probed in $3 \times$ folding buffer (333 mM HEPES-KOH pH 8, $333 \mathrm{mM} \mathrm{NaCl}, 33.3 \mathrm{mM}$ $\mathrm{MgCl}_{2}$ ) in the presence or absence of SRP14, HMGB3 or PTB recombinant protein at 5, 10 and 20 protein/RNA molar ratio (0.41-1.6 $\mu \mathrm{M})$. RNP complexes were formed by incubation for $20 \mathrm{~min}$ at room temperature then probed with 1-methyl-7nitroisatoic anhydride (1M7, $65 \mathrm{mM}$ in DMSO) or DMSO for $4 \mathrm{~min}$ at $37^{\circ} \mathrm{C}$, then recovered by ethanol precipitation. Primer extension was conducted as described previously with $0.4 \mu \mathrm{M}$ fluorescently labeled odp3102 primer (6-FAM or HEX, SigmaAldrich, Supplementary Table 2). The dideoxy sequencing reactions were generated using unmodified RNA, labeled primers (PET or NED, Applied Biosystems) and $0.5 \mathrm{mM}$ ddGTP. cDNAs were recovered by ethanol precipitation and separated by capillary electrophoresis with LIZ500 size standard (ABI 3130, AGRF). Data was processed using the QuShape software (Karabiber et al., 2013). Protections induced by SRP14, HMGB3 or PTB binding were indicated by a reduction in the normalized SHAPE reactivities.

\section{RESULTS}

\section{Detection of 243 Putative Tat-RNA and Cellular Protein Interactions}

To explore MS RNA binding partners during latency, we initiated a proteomic approach based on affinity chromatography purification of RNA-protein complexes (Maenner et al., 2010; Bar et al., 2011) formed upon incubation of in vitro transcribed tat RNA with protein lysate, followed by protein identification by mass spectrometry. An overview of the processes is shown in Figure 1A. Protein lysates were prepared from the HIV1 latently infected $\mathrm{T}$ cell line, J-Lat6.3 where the cells were either left untreated (latent infection) or activated with TNF$\alpha$ (productive infection). The RNA of interest, 5'UTRtat1-Tat and Tat with three binding sites for the MS2 coat protein fused at their $3^{\prime}$-ends were used as baits. In parallel, a control RNA that only contained the three MS2 binding sites was used. RNA 
A

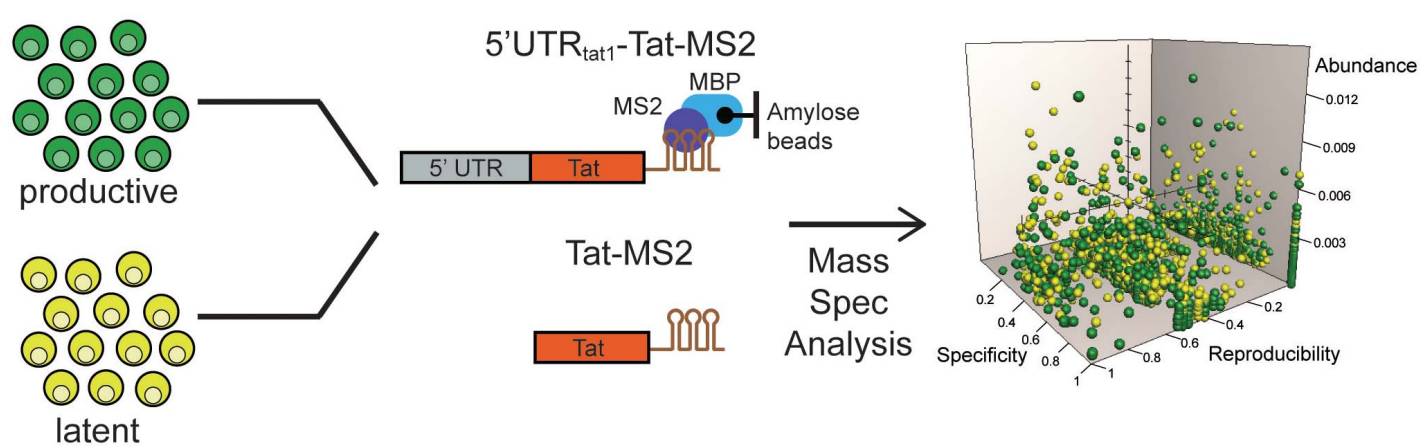

B

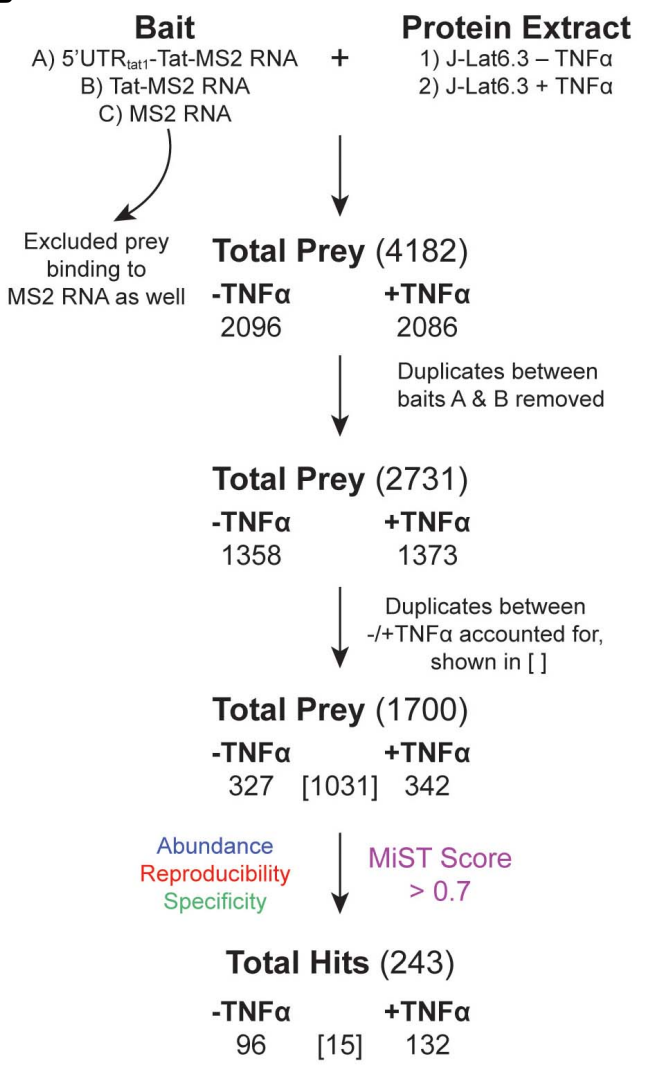

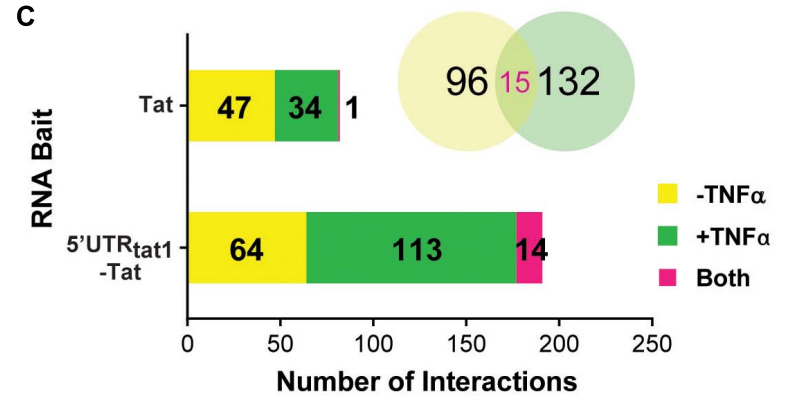

D

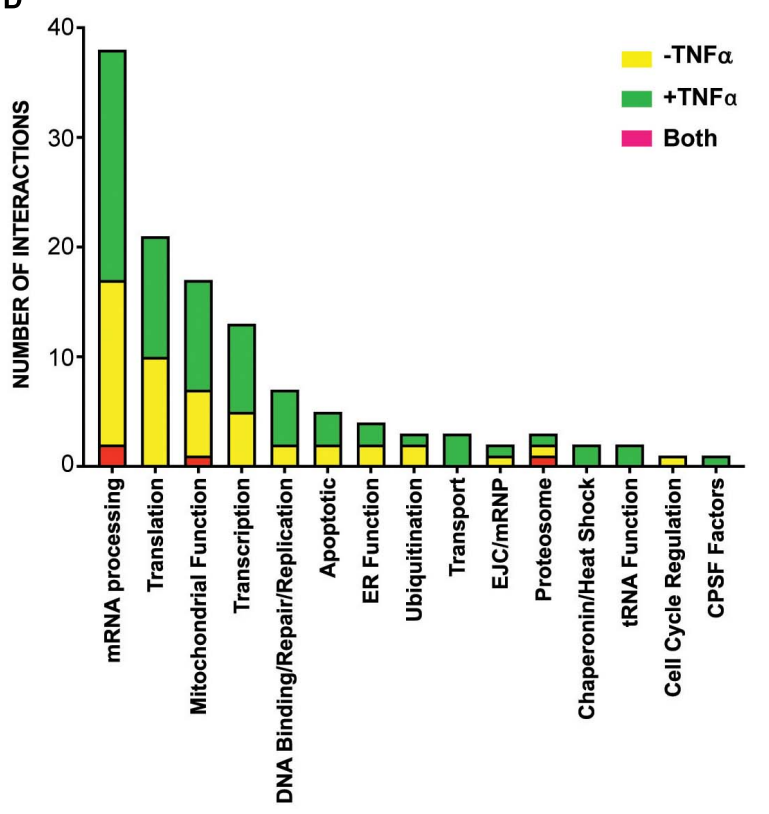

FIGURE 1 | Enrichment in mRNA processing and translation factors assembling on tat mRNA. (A) Representation of the affinity purification strategy used for isolation of cellular RNA binding proteins that interact with tat mRNA. Whole cell lysates were prepared from the latently infected T-cells, J-Lat clone 6.3 where untreated cells were used as the latent sample and TNF- $\alpha$ activated cells were used as the productively infected sample. Cellular proteins that interact with tat mRNA were pulled down through MBP-MS2 affinity purification using two RNA baits that contained Tat exon 2 with or without the $5^{\prime} U$ TR from tat 1 mRNA and fused to three MS2 sequences. Eluted RNA-protein complexes were analyzed by mass spectrometry. MiST analysis was used to determine the most biologically relevant interactions after combining data from three replicates. (B) The filtering steps applied to refine the mass spectrometry data. Prey binding to the control bait, MS2 RNA were excluded, followed by removal of duplicate prey captured by baits A (5'UTRtat-Tat) and B (Tat RNA). Prey were then defined as derived from uniquely -TNFa or +TNFa lysates or from both. Lastly, MiST analysis was applied to score interactions and a confidence threshold of 0.7 was used to refine the list of candidates for relevant Tat mRNA: cellular protein interactions. (C) Breakdown of the 243 proteins selected by MiST analysis by bait and lysate. (D) Annotation of the 243 proteins based on GO biological process. 
retention to amylose beads was mediated by the MS2-MBP fusion protein containing the MS2 coat protein RNA binding domain and the E. coli maltose binding protein (MBP). RNP complexes were eluted by maltose and the protein composition of the purified RNPs were analyzed by mass spectrometry. The proteins identified by MS were quantitatively scored using the mass spectrometry interaction statistics (MiST) platform devised by Jäger et al. (2011).

Several filtering steps described in Figure 1B were applied to refine the protein preys obtained by MBP-MS2 pull-down for generation of a list of 1,700 unique proteins where duplicates between the different conditions were accounted for. These 1,700 proteins were quantitatively scored based on their abundance, reproducibility and specificity and 243 putative Tat RNA:protein interactions were identified using a confidence threshold of 0.7 for biological relevance (Figure 1B).

As expected, when assigned to their respective bait (Figure 1C, bar graph) or protein lysate (Figure 1C, Venn Diagram), we observed a larger number of proteins interacting with the longer RNA bait (191 proteins interacting with the 5'UTRtat1-Tat-MS2 vs. 82 with Tat-MS2). Moreover, a higher number of interactions were detected with the lysates prepared from activated T-cells (147 proteins for $+\mathrm{TFN} \alpha$ vs. 111 proteins for $-\mathrm{TNF} \alpha$ ). Analysis of the 243 proteins by broad gene ontology terms (Spliceosome database, Cvitkovic and Jurica, 2013) showed that the top two overrepresented annotated biological processes were mRNA processing and translation (Figure 1D). Analysis of the GO molecular function also showed a predominance of RNA-binding proteins (Supplementary Figure 1).

\section{Knockdown of Tat RNA Binding Proteins Affect Latent and Productive Infection of HIV-1}

From the 243 proteins identified through MS2 chromatography affinity purification, thirteen proteins were selected to follow through with validation studies (Figure 2A and Supplementary Table 3). The main criteria for selection were the MiST score, the role of the protein in cellular pathways and the novelty of the protein in the context of regulation of HIV-1 latent infection. Two additional proteins, PTB1 and HSP90A were selected as controls as both proteins have previously been shown to be involved in regulation of HIV-1 latency (Lassen et al., 2006; Anderson et al., 2014).

For further refinement of the list of candidates for in-depth investigation, we assessed the effect of knockdown of the proteins on HIV-1 latent and productive infection. Jurkat T-cells were transduced with shRNA expressing lentivectors targeting each of the fifteen protein targets for knockdown (KD). MCM5 KD induced high cell death hence MCM5 was excluded from further investigation. Successfully transduced cells were sorted into a bulk population by $\mathrm{RFP}^{+}$expression (Supplementary Figure 2A) where the degree of knockdown was heterogeneous between individual cells. Success of the knockdown of the gene targets in the bulk Jurkat cell populations were confirmed by western blot and densitometry analysis (Supplementary Figure 2B). To assess the outcome of protein knockdown on HIV-1 infection, we used a single-round dual-fluorescent reporter virus (DuoFluo, R7GEmTB) based on the R7GEmC backbone described in Calvanese et al. (2013). In our DuoFluo virus, HIV-1 5'-LTR controls eGFP expression indicative of productive infection and mTagBFP2, controlled by the EF1 $\alpha$ promotor, is the marker of latent infection (Figure 2B). The fluorescent phenotype of cells and corresponding profile of HIV1 infection are shown in the grid (Figure 2B). We confirmed the ability of the eGFP/mTagBFP2 expressing dual-fluorescent reporter virus to identify the presence of latently (blue, $\mathrm{BFP}^{+}$) and productively (green, $\mathrm{GFP}^{+}$or cyan, $\mathrm{GFP}^{+} \mathrm{BFP}^{+}$) infected Jurkat and primary $\mathrm{CD} 4^{+}$T-cells by fluorescence microscopy after infection with R7GEmTB (Supplementary Figure 3).

All bulk populations of the $\mathrm{KD} \mathrm{RFP}^{+}$Jurkat cells were infected with the DuoFluo virus and collected for flow cytometry analysis after $72 \mathrm{~h}$. Levels of eGFP and mTagBFP2 expression were assessed by gating on the $\mathrm{RFP}^{+}$population and compared against DuoFluo infected untransduced RFP- parental Jurkat cells. An increase in productive infection was detected after knockdown of FLNA, HMGB3, PTBP1, HSP90AA1, and KIF2C (Figure 2C, green bars). On the other hand, a dramatic increase in latent infection was seen after knockdown of TOP2A, SRP14, HNRNPH1, DDX1, and HNRNPL (Figure 2C, blue bars). The increase in latent infection after knockdown of TOP2A and HNRNPH1 was coupled with a decrease in productive infection.

PTB, known to facilitate the export of multiply spliced (MS) mRNAs to the cytoplasm (Lassen et al., 2006), appears to play a role in controlling latent and productive infection, as increases in both forms of infection were observed after knockdown of the protein (productive: $70.9 \%$, latent: $7.61 \%$ ). However, the effect on latent infection was smaller than expected. Interestingly, two genes, SRP14 and HMGB3 have not been previously reported to play a role in the regulation of HIV-1 replication and their knockdown here had very marked effects on latent and productive infection, respectively. Knockdown of SRP14 had no effect on productive infection but increased dramatically the percentage of cells entering into latency (17.0\% vs. Jurkat $1.71 \%$, Figure 2D). In contrast, knockdown of HMGB3 had no effect on latent infection, but increased the percentage of productively infected cells $(88.8 \%$ vs. Jurkat $65.2 \%$, Figure $2 \mathrm{D})$. These data suggest that SRP14 is a negative regulator of latent infection, whilst HMGB3 is a negative regulator of productive infection.

\section{Knockdown of SRP14 and HMGB3 Strongly Modulates Splicing at SA3}

As a strong block to multiply splicing of HIV-1 mRNA was recently characterized in $\mathrm{CD}^{+}{ }^{+}$T-cells isolated from patients under ART (Yukl et al., 2018), we examined the role of knockdown of the RNA binding proteins on tat mRNA splicing using an HIV-1 splicing reporter, which harbors SD1 5'ss and SA3, SA4a,b,c and SA5 3'ss (Ropers et al., 2004). In this splicing reporter construct, Tat-exon1,2 (nt 1-839/5590-6044 NL4-3) was placed in the context of a human gene to recapitulate HIV integration in latently infected cells. The context of HIV-1 integration in the latent cell line, ACH2 cells (Clouse et al., 1989, Folks et al., 1989) was used as the basis of the design hence NT5C3 
A

\begin{tabular}{|c|c|c|c|}
\hline \multicolumn{2}{|c|}{$-T N F \alpha$} & $+\mathrm{TNF} \alpha$ & Controls: \\
\hline $\begin{array}{l}\text { KIFC1 } \\
\text { TOP2A } \\
\text { HMGB3 }\end{array}$ & $\begin{array}{l}\text { DDX1 } \\
\text { KIF2C } \\
\text { MCM5 }\end{array}$ & $\begin{array}{ll}\text { hnRNP L } & \text { MAP4 } \\
\text { hnRNP U } & \text { SRP14 } \\
\text { hnRNP H } & \text { RBBP7 }\end{array}$ & $\begin{array}{c}\text { Known regulators of HIV } \\
\text { latent and/or } \\
\text { productive infection }\end{array}$ \\
\hline & & FLNA & PTB1 HSP90A \\
\hline
\end{tabular}

B

R7GEmTB

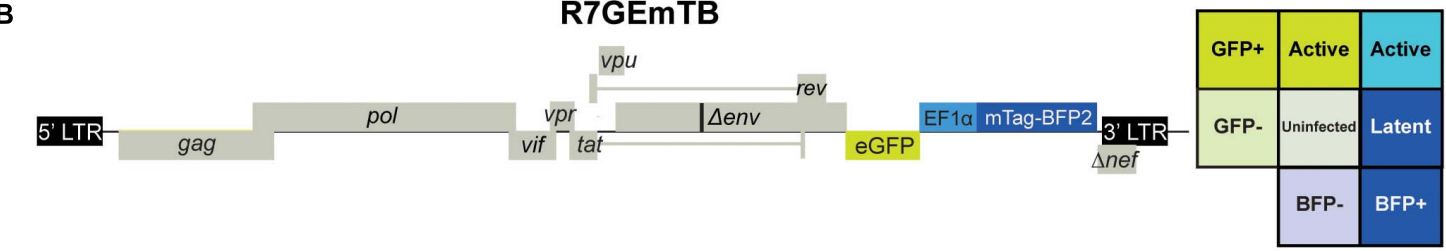

C

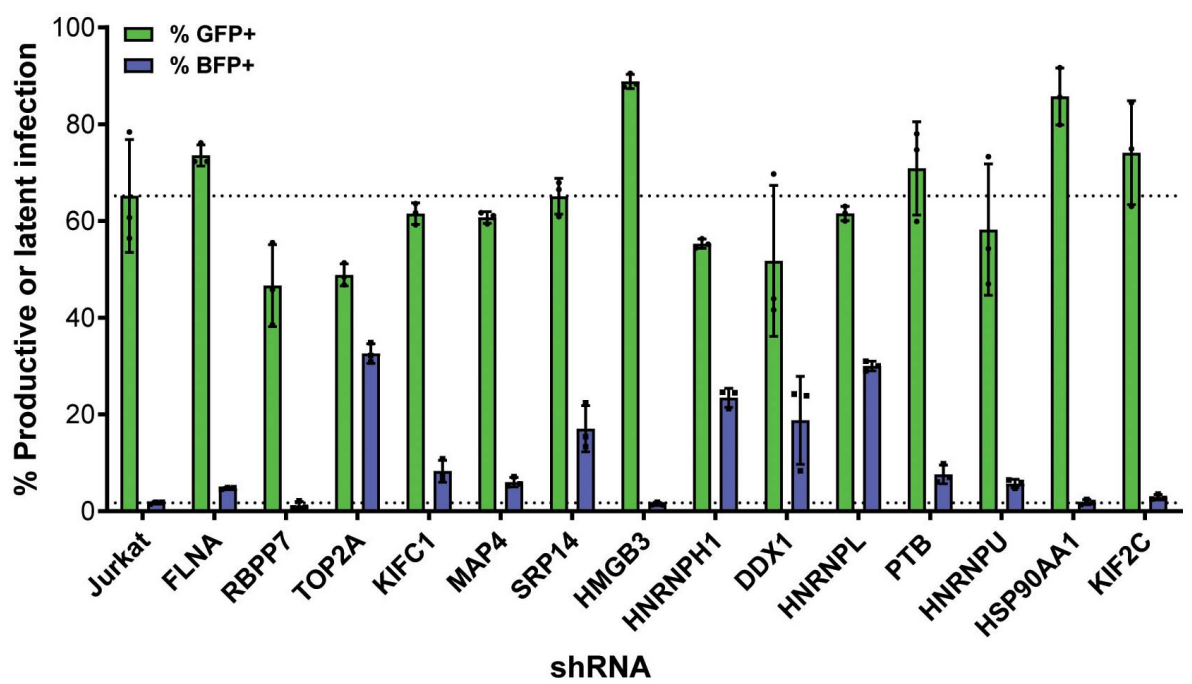

D

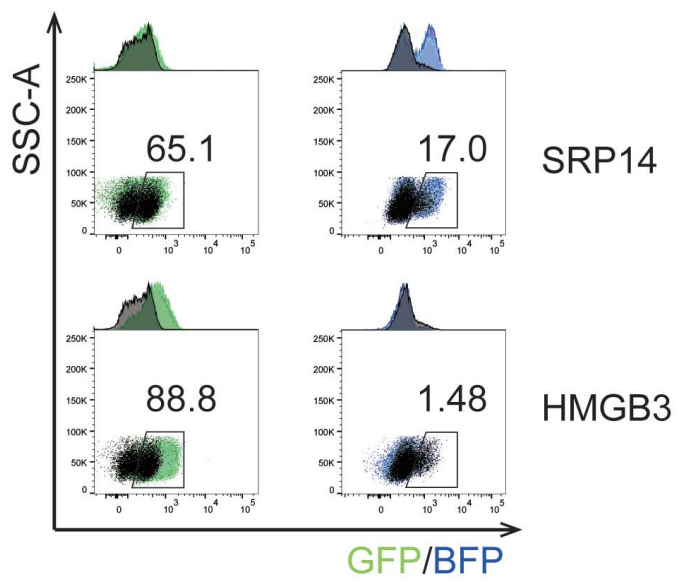

FIGURE 2 | SRP14 and HMGB3 knockdown impact HIV latent and productive infection. (A) Short-list of 13 proteins chosen for follow-up validation and two control proteins, PTB and HSP90A that are known regulators of HIV-1 replication. (B) Genome of the single round DuoFluo virus [R7/E-/GFP-EF1 $\alpha$-mTagBFP2 (R7GEmTB)] used in this study. The fluorescence profiles that correspond to latent or productive infection are shown in the grid. (C) Bulk populations of RFP+ shRNA knockdown (KD) Jurkats were infected with the single round DuoFluo virus pseudotyped with the dual-tropic 92HT593.1 envelope, and collected $48 \mathrm{~h}$ later for flow cytometry analysis. Percentage of productive or latently infected cells after knockdown of the specific protein is shown, with productive infection in green and latent infection in blue. Data shown are means of three independent experiments \pm SEM. (D) Scatter plots highlighting the shift in GFP ${ }^{+}$or BFP ${ }^{+}$populations after infection of RFP+ SRP14 (top) or HMGB3 (bottom) KD Jurkats with DuoFluo virus. Black population represent infected, untransduced Jurkat cells, while green/blue populations represent infected SRP14/HMGB3 KD RFP+ Jurkats. Values represent the percentage of GFP+ or BFP+ cells in KD RFP+ Jurkat cells. 
exon 5 and intron 5 were incorporated upstream of the HIV-1 $5^{\prime}$-LTR (Figure 3A). Renilla luciferase (LucR) was introduced at the $3^{\prime}$ end of Tat exon 2, thus an increase in LucR would indicate splicing at SA3, SA4a,b,c or SA5. Co-transfection of the splicing reporter with an LTR-LucF reporter cassette allows Firefly luciferase to be used as a specific readout of splicing at SA3 and Tat production. The effect of cellular proteins on use of SA3 in the $\mathrm{Tat}^{+}$splicing reporter were compared to a matched Tatsplicing reporter lacking both the HIV introns and Tat exon 2 (Figure 3A). An increase in LucR expression in the Tat- context shows the promotion of splicing between the cellular splice site, SDc site at the $3^{\prime}$ end of NT5C3 exon 5 and the SAc upstream of the $5^{\prime}$-LTR.

Bulk populations of the KD RFP + Jurkat cells were cotransfected with the $\mathrm{Tat}^{+}$or Tat- splicing reporter and the LTRLucF cassette and harvested for luciferase analysis $24 \mathrm{~h}$ later. The LucF/LucR ratio was calculated for both the Tat ${ }^{+}$and Tatcontexts in response to the knockdown of each protein target. Fold changes in Tat splicing over transfected RFP- untransduced Jurkat cells were reported in Figure 3B. Knockdown of SRP14 decreased splicing at SA3 by sixfold compared to untransduced Jurkats, whereas knockdown of HMGB3 and PTB increased the use of SA3 by 8.9 - and 8.8-fold, respectively (Figure 3B). None of the other ten proteins of interest affected splicing at SA3. The effect of SRP14 and HMGB3 KD on use of SA3 is consistent with the effects of these proteins on latent and productive infection, suggesting an important role of these proteins in Tat mRNA processing and regulation of HIV-1 infection.

\section{Knockdown of SRP14 and HMGB3 Impacts Tat Expression and Function}

Major blocks of HIV transcription and translation have been reported during latency (Khoury et al., 2018a). Due to the central role of Tat protein in promoting HIV transcription and post-transcriptional events, this warranted a deeper investigation of the role of SRP14, HMGB3 and PTB on Tat expression. One caveat of using bulk populations of the KD $\mathrm{RFP}^{+}$ Jurkat cells is the large clonal variation. To circumvent this obstacle, single clones were sorted following 12 days of puromycin selection and assessed through expression of RFP by flow cytometry (Supplementary Figure 2C). To examine $\mathrm{KD}$ efficiency in the various clones, changes in mRNA levels compared to untreated Jurkat T-cells was determined by RTqPCR. Importantly, we observed across all single clones tested a significant reduction in the levels of SRP14, HMGB3 and PTB mRNA compared to untransduced Jurkats (FC vs. Jurkat $\geq 50 \%$, Supplementary Figure 2D).

Next, bulk and single SRP14 (B5, C10, and G4), HMGB3 (C2, D4, E7, and G9) or PTB (C10, C11, D5, and D8) shRNA

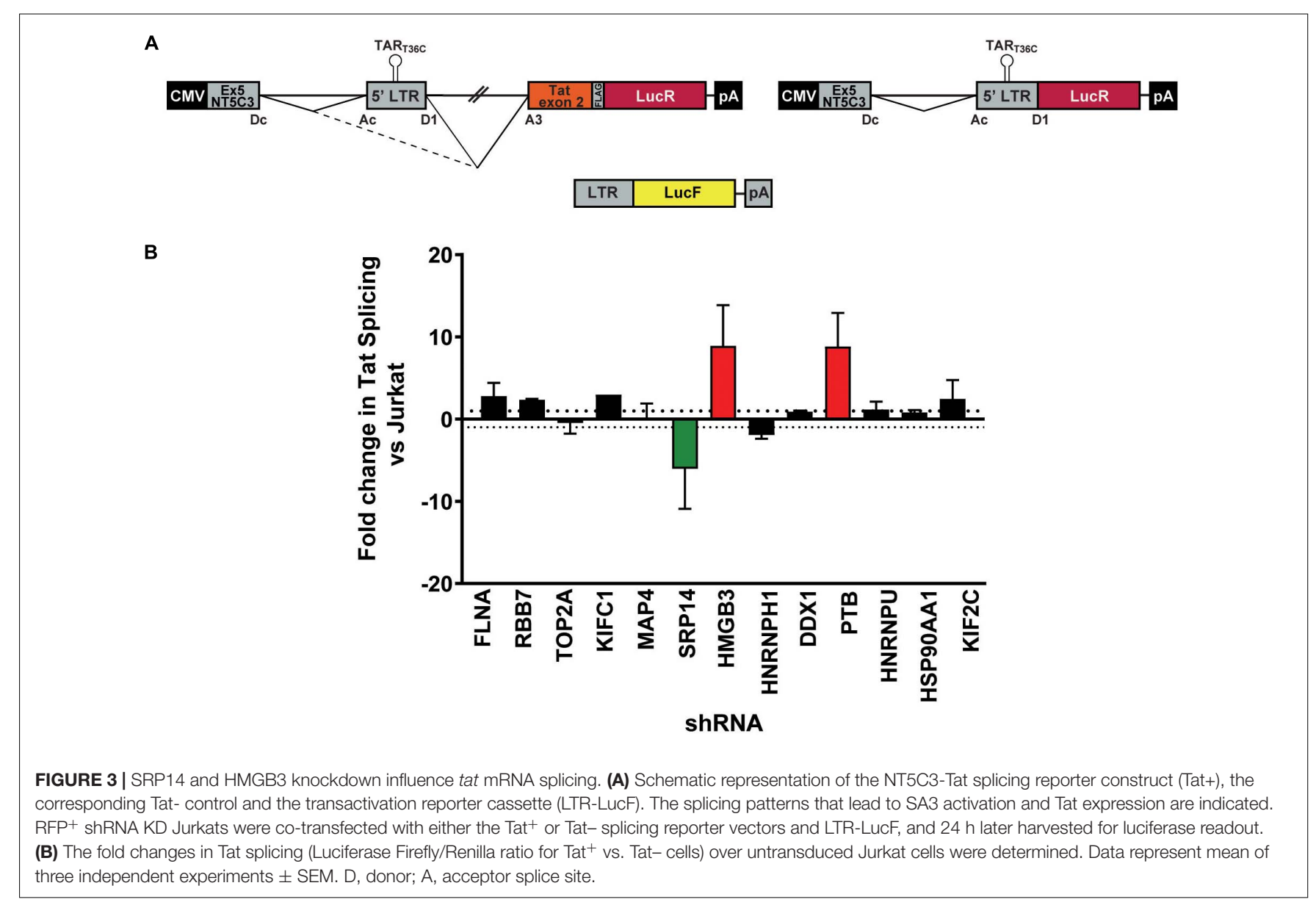


KD clones were transfected with Tat expression constructs to assess the effect on Tat transactivation and translation. We previously characterized a highly conserved element underlying the Tat open reading frame, named TIM-TAM (for Tat IRES modulator of tat mRNA) and characterized its role in controlling Tat translation through cap- and IRES-dependent mechanisms (Khoury et al., 2020). Moreover, we developed a model system to assess Tat cap and IRES translation (Nguyen et al., 2019).
In our IRES-dependent Tat translation expression cassette, the Tat encoding exons have been incorporated into the human growth hormone gene (GH1), where readthrough transcription and alternative splicing would allow low level expression of GH1Tat protein through an IRES-dependent mechanism (Figure 4A). In the control construct, Tat was placed under the control of a cytomegalovirus (CMV) promoter allowing Tat expression in a cap-dependent manner. In both contexts, Tat was cloned in phase

A

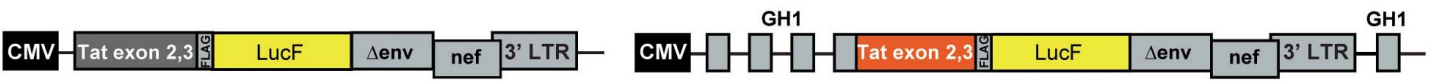

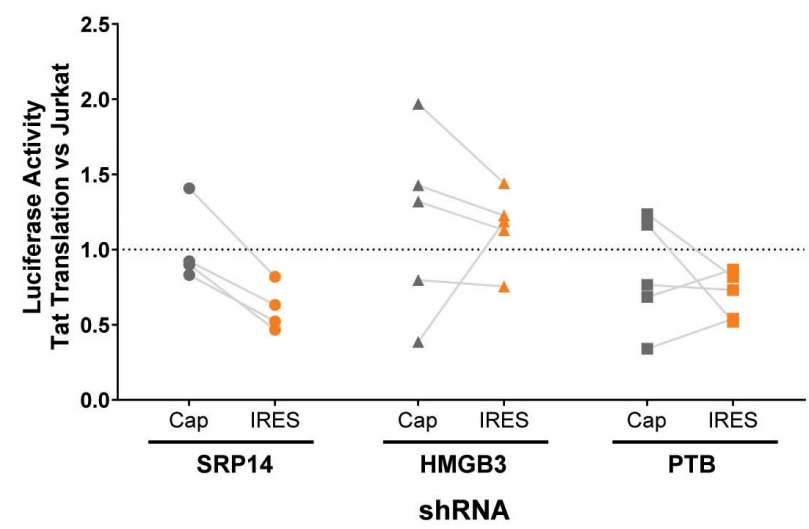

B
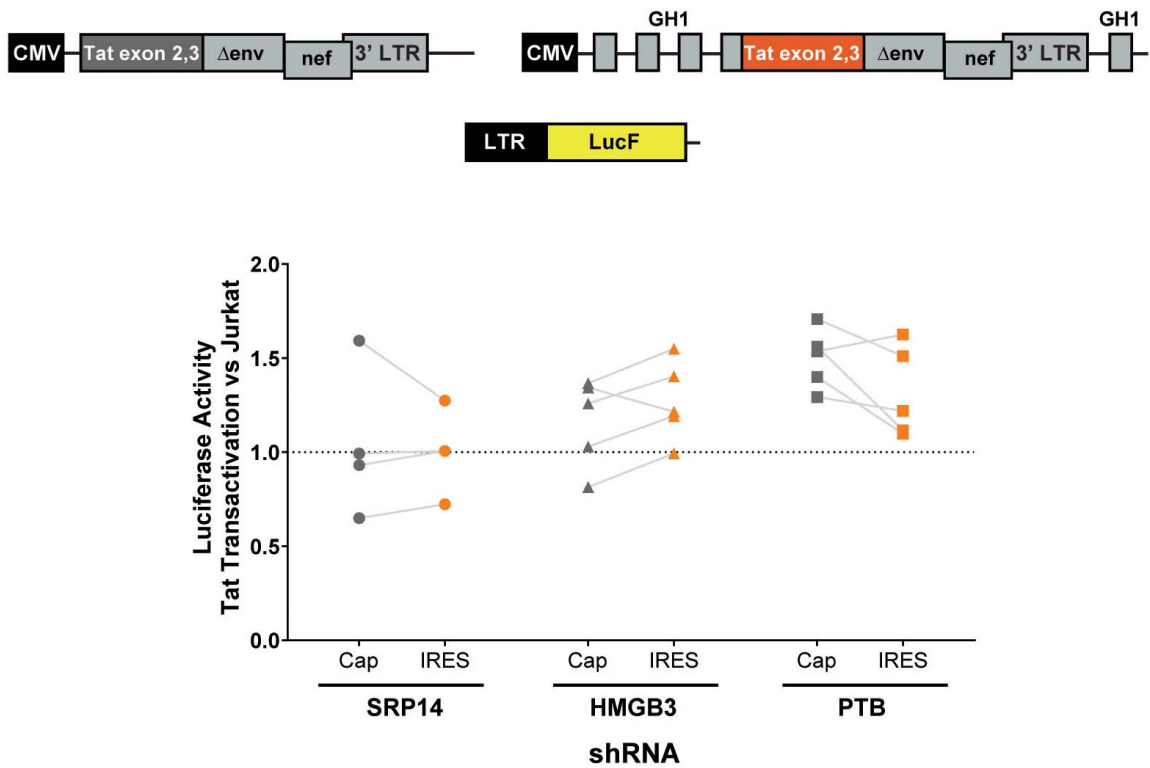

FIGURE 4 | SRP14 and HMGB3 knockdown control Tat -cap and -IRES dependent translation. Diagrams depicting Tat reporter constructs used to study Tat cap(left) or IRES- (right) dependent translation (A) and transactivation (B). Luciferase Firefly expression, which is produced in fusion with Tat (in A) or under the control of the $5^{\prime}$-LTR (in B), is used as a readout for Tat translation and transactivation, respectively. Bulk populations or single clones selected for knockdown of SRP14, HMGB3 or PTBP1 were transfected with Tat cap or IRES expression constructs to assess the effect on Tat translation (A) or transactivation (B). Cells were harvested for luciferase analysis $24 \mathrm{~h}$ later. Firefly luciferase activity (LucF) was normalized on Renilla luciferase activity (LucR) and shown as a fold change over untransduced Jurkats. Data shows mean of two independent experiments with single clones and bulk populations. CMV, cytomegalovirus promoter; GH1, human growth hormone gene. 
with LucF hence lucF expression is a marker of Tat translation. Bulk population and single clones of the $\mathrm{KD} \mathrm{RFP}^{+}$Jurkat cells were transfected with cap or IRES Tat-LucF expression constructs and harvested for luciferase analysis $24 \mathrm{~h}$ later. Fold changes in luciferase activity were then calculated in comparison to RFPuntransduced Jurkats. In the SRP14 KD RFP + Jurkats, Tat translation was reduced from the cap-dependent context in the bulk population and 2 of 3 single clones and from the IRESdependent context in the bulk population and all three single clones (Figure 4A). In contrast, HMGB3 knockdown induced an increase in Tat translation from the cap-dependent context for the bulk population and 2 of 4 single clones, and in the bulk population and 3 of 4 single clones for the IRES-dependent context (Figure 4A). Lastly, knockdown of PTB resulted in a decrease in Tat translation in 2 of 4 single clones transfected with Tat-lucF construct, as well as all 4 single clones and the bulk population transfected with Tat IRES construct (Figure 4A).

We next investigated the effect of SRP14 and HMGB3 $\mathrm{KD}$ on Tat transactivation by using a modified expression cassette system, where Tat is translated through an IRESor cap-dependent pathway and the luminescence readout is induced by Tat transactivation of an HIV-1 LTR-LucF reporter cassette. The effect on Tat transactivation after knockdown of SRP14 was variable across the single clones for both cap- and IRES-Tat translation, however, there was a clear increase in Tat transactivation for 3 out of 4 Tat-cap transfected single clones and 4 out of 4 Tat-IRES transfected single clones after knockdown of HMGB3 compared to untransduced Jurkats (Figure 4B). Knockdown of PTBP1 resulted in an increase in Tat transactivation for all single clones transfected with Tat-Cap and Tat-IRES (Figure 4B). These data demonstrate a role of SRp14 and HMGB3 in controlling HIV-1 latent and productive infection in Tat-dependent manner.

\section{SRP14 and HMGB3 Binds in the Vicinity of Tat Start Codon}

Whilst SRP14 and HMGB3 were detected in the RNP complexes (Supplementary Figure 4), further investigation was required to assess direct interaction of these proteins with tat mRNA. To delineate SRP14, HMGB3, and PTB binding sites on multiply spliced RNA, we performed footprinting assays coupled to SHAPE (selective 2' hydroxyl acylation analyzed by primer extension) analysis. We have recently determined tat 1 and tat 2 mRNA folding using enzymatic and chemical probing and identified a highly conserved sequence-structure within MS RNA, TIM-TAM (Khoury et al., 2020). TIM-TAM forms the apical part of an irregular stem-loop structure $\mathrm{SLS}_{\mathrm{A} 3}$ that harbors the Tat start codon. TIM-TAM controls the timing and level of Tat translation during the early and late phases of infection, while promoting latent infection and virus reactivation. Footprinting assays were performed on RNP complexes formed by tat 2 transcript (Figure 5A) and recombinant proteins at three different $[\mathrm{RNA}] /[$ protein] ratios 5, 10, and 20. Normalized shape reactivities and probing data were used to determine the binding sites of SRP14, HMGB3, and PTB. At the lowest [RNA]/[protein] ratio, protections were mainly detected on $\mathrm{SLS}_{\mathrm{A} 3}$ including
TIM-TAM and its bordering sequences. Strong protections were also observed in the $5^{\prime}$ untranslated region, more specifically on the TAR, PBS, and DIS elements (Figure 5B). Upon increasing SRP14, HMGB3, and PTB concentration, protections of TIMTAM were reinforced and new ones were detected on the Tat start codon and in the vicinity of SA3. Altogether, these data are consistent with direct binding of SRP14, HMGB3 as well as PTB to MS RNA highlighting a potential role of these RNA binding proteins in controlling Tat expression during latent infection.

\section{SRP14 Reactivates HIV-1 Latently Infected Cells and Virus Production}

To assess the role of SRP14 in controlling latent infection, we tested the effect of its overexpression on virus reactivation in a T-cell line model of latent infection, using the J-Lat 10.6, 8.4 and A2 clones. J-Lats are Jurkat derived cells containing one stably integrated, but transcriptionally silenced full-length HIV1 genome with GFP in place of the nef gene (Jordan et al., 2003). J-Lat cells were transduced with pInducer-SRP14-T2AmtagBFP2 lentiviral vectors and cultured in the absence or presence of doxycycline ( + Dox) for 2 days. Representative scatter plot highlighting mtagBFP2 expression following treatment of transduced cells with $5 \mu \mathrm{g} / \mathrm{mL}$ doxycycline is shown in Figure 6A. SRP14 expression was validated by western-blot analysis (data not shown). The different J-Lat clones exhibit variable levels of basal GFP expression. Upon doxycycline treatment, we observed an increase in GFP expression in all $\mathrm{T}$-cell lines. Indeed, a significant increase in mean fluorescence intensity (MFI) was detected for $\mathrm{BFP}^{+} \mathrm{GFP}^{+}$cells vs. $\mathrm{GFP}^{+}$cells alone (Figure 6B).

To determine whether differences in RNA binding protein levels in resting and activated $\mathrm{CD} 4^{+} \mathrm{T}$-cells might be involved in the observed effect on HIV expression, we measured SRP14, HMGB3 and PTB protein and RNA levels in resting and $\alpha$-CD3/CD28 stimulated CD4 ${ }^{+}$T-cells from patients living with HIV on ART. Western-blot analysis indicated low expression levels of SRP14 and HMGB3 in resting cells, and upon stimulation a 2.5- and 18.8-fold increase in SRP14 and HMGB3 protein level was detected, respectively (Figure 7A). Similar results were observed with RT-qPCR analysis of HMGB3 mRNA levels between resting and activated $\mathrm{CD} 4^{+} \mathrm{T}$-cells as we detected a 28.5-fold increase in $H M G B 3$ mRNA expression following $\mathrm{CD}^{+}{ }^{+} \mathrm{T}$-cell activation (Figure $7 \mathrm{~B}$ ). However, no changes in SRP14 mRNA levels were seen in response to T-cell activation. Interestingly, while a 4.4-fold increase in PTB mRNA expression was observed, no significant change in PTB protein expression was detected following stimulation of $\mathrm{CD} 4^{+} \mathrm{T}$-cells.

PTB was identified as an HIV RNA binding protein that induces virus reactivation and release of replication competent virus in resting $\mathrm{CD}^{+}{ }^{+} \mathrm{T}$-cells from patient on ART (Lassen et al., 2006). To examine whether SRP14 might also act as a positive factor for HIV-1 gene expression, resting $\mathrm{CD}_{4}^{+} \mathrm{T}$-cells isolated from people living with HIV on ART were electroporated with SRP14 or PTB Dox-inducible expression constructs alone or in combination using an Amaxa nucleofector. After 48 h, virion release into the culture supernatant was assessed using 
A

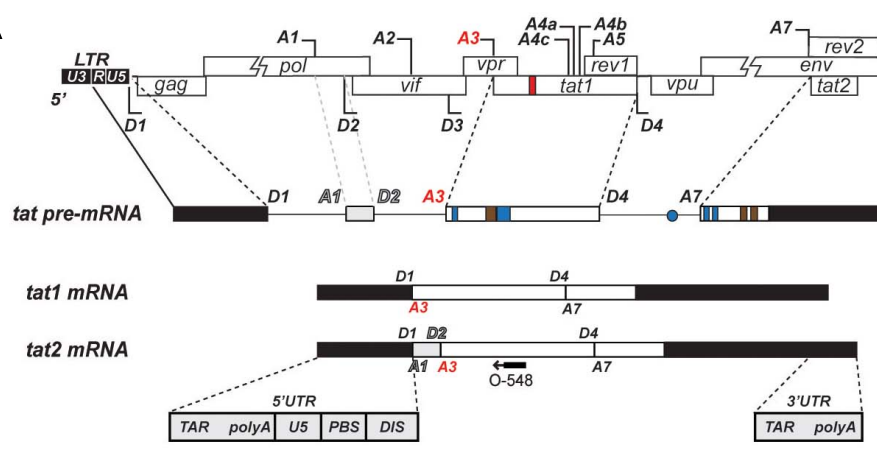

B

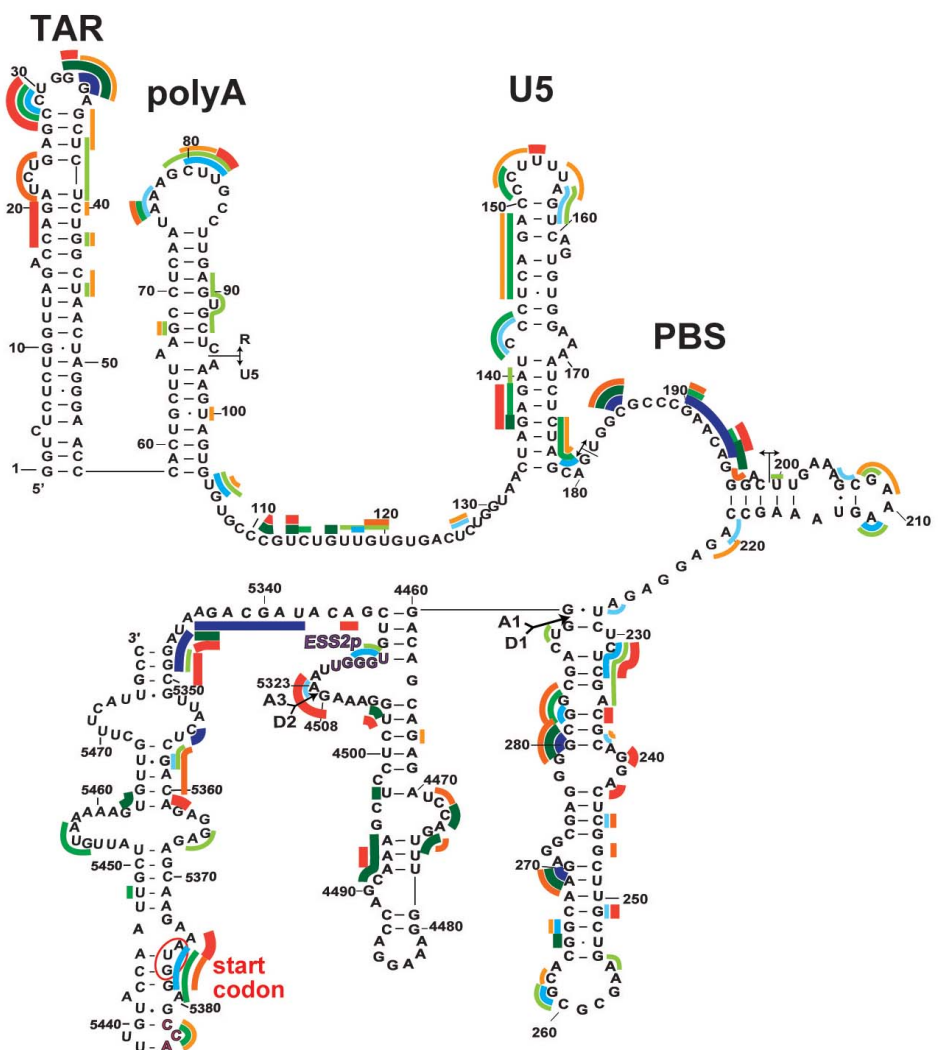

DIS

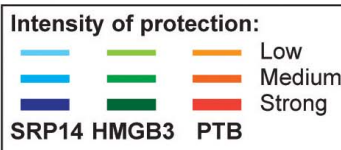

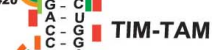

$\left(\begin{array}{cc}A-A \\ C G\end{array}\right)$ ( $)$

$S L S 3_{A 3}$

FIGURE 5 | Interaction of SRP14, HMGB3 and PTB with tat mRNA. (A) Schematic representation of HIV-1 proviral genome, tat pre-mRNA and multiple splice events leading to tat1 and tat2 mRNAs production during the early phases of HIV-1 infection. D represent donor splice sites, A represent acceptor splice sites, $5^{\prime}$ UTR/3'UTR indicate $5^{\prime} / 3^{\prime}$ untranslated regions. The hybridisation site used for primer extension with O-548 (also known as Odp3102) is indicated by black arrow. (B) Probing of SRP14, HMGB3, and PTB binding sites on tat2 mRNA. Tat2 transcript (1 pmol) was modified with $1 \mathrm{M} 7$ ( $65 \mathrm{mM}$ ) in the absence or presence of increasing concentration $(0.41-0.83-1.6 \mu \mathrm{M})$ of recombinant proteins. Conditions of modification are given in Materials and Methods. Protections generated by SRP14, HMBG3 and PTB recombinant proteins are indicated on the secondary structure model of tat2 mRNA by blue, green and red lines, respectively. Pale, medium, and dark colors indicate the intensity of protections (low, medium and strong protections). Numbering of nucleotides and positions of the cis regulatory elements are given in reference to HIV-1 BRU (K02013). The start codon of Tat protein is circled. 

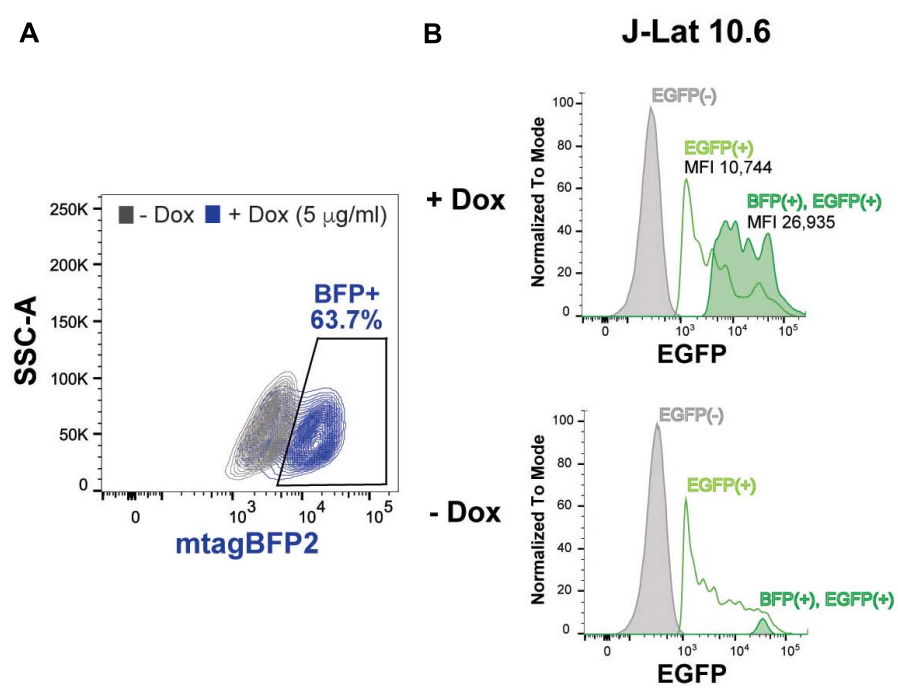

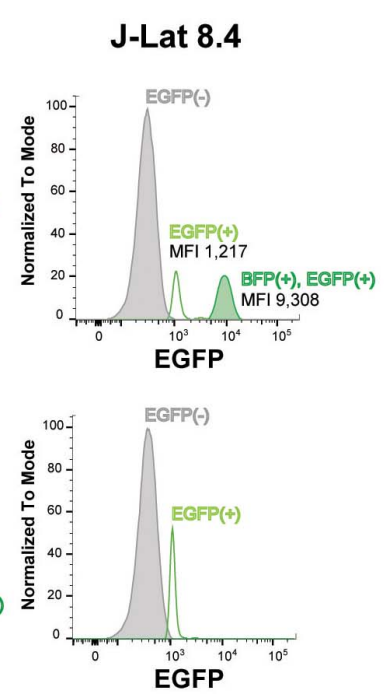

FIGURE 6 | SRP14 expression reactivates latently infected T-cell lines. (A) J-Lat cells were transduced with SRP14-T2A-mtagBFP2 lentivector and cultured with (+Dox) or without (-Dox) doxycycline at $5 \mu \mathrm{g} / \mathrm{mL}$. Cells were analyzed for mtagBFP2 expression by flow cytometry $72 \mathrm{~h}$ post-infection. (B) Histogram depicting EGFP $^{+}$expression in latently infected T-cells following transduction with SRP14-T2-mtagBFP2. Virus reactivation in J-Lat 10.6, 8.4 and A2 clones is shown by an increase in mean fluorescence intensity $(\mathrm{MFI})$ of $\mathrm{BFP}^{(+)} \mathrm{EGFP}^{(+)}$cells in comparison to $\mathrm{EGFP}^{(-)}$and EGFP(+) cells.

an RT-ddPCR assay. One out of the four patient CD4 ${ }^{+}$T-cells electroporated with SRP14 and SRP14 ${ }^{+}$PTB presented an upregulation of virus production upon doxycycline treatment (Figure 7C). Virus production following SRP14 overexpression was not coupled with an increase in cell activation as only $0.9 \%$ and $1.3 \%$ of SRP14 transfected cells expressed middle (CD25) and late (HLA-DR) activation markers, respectively (Figure 7D).

\section{DISCUSSION}

We have characterized tat RNA:cellular protein interactions differentially expressed between productive and latent infection. Out of the 243 proteins identified by mass spectrometry, multiple cellular factors were investigated for their putative roles in the control of Tat expression and viral replication. A consistent effect on Tat expression and HIV-1 replication was exerted by both SRP14 and HMGB3, where SRP14 acts as a positive regulator of Tat expression and negative regulator of latent infection while HMGB3 acts as a negative regulator of Tat expression and negative regulator of productive infection (Figure 8). However, the exact mechanisms exerted by SRP14 and HMGB3 on the pathways of Tat expression have not been determined. It should be noted that knockdown of SRP14 and HMGB3 affected to a larger degree Tat expression in the IRES context. This suggests that SRP14 and HMGB3 proteins are acting through the Tat IRES pathway, by directly interacting with TIM-TAM or by acting as scaffolds for other RNA-binding proteins.

In a recent study, Yukl's group identified HIV multiple splicing as a common block in three primary cell models of latent infection and in peripheral $\mathrm{CD}^{+}{ }^{+} \mathrm{T}$-cells isolated from HIV infected ART suppressed individuals (Moron-Lopez et al., 2020), confirming previous findings from the same group that showed a series of blocks to HIV proximal elongation, distal transcription/polyadenylation and splicing preventing HIV expression in $\mathrm{CD}^{+}{ }^{+} \mathrm{T}$-cells from blood of HIV infected patients on ART (Yukl et al., 2018). Prior studies have shown that MS RNA encoding Tat protein inhibits the establishment of HIV latency (Donahue et al., 2012). When present, Tat activates virus replication at a higher rate than any of the known LRAs (Razooky et al., 2015; Khoury et al., 2018b) by potently inducing HIV transcription and splicing (Khoury et al., 2018b). Moreover, by controlling its own production at the splicing (Jablonski et al., 2010) and translational levels (Charnay et al., 2009, Khoury et al., 2020), Tat acts as a switch for productive and latent HIV infection (Khoury et al., 2020).

siRNA knockdown of TOP2A in Sup T1 cells (Balakrishna et al., 2013), MAP4 in TZM-bl, HEK 293T and HeLa P4.2 cells (Brass et al., 2008; König et al., 2008; Gallo and Hope, 2012), HNRNPH1 in 293T cells (König et al., 2008), DDX1 in HeLa cells (Edgcomb et al., 2012) and HNRNPU in HeLa P4/R5 cells (Zhou et al., 2008) inhibit HIV-1 replication, corroborating our findings following shRNA KD of these proteins in Jurkat cells. Furthermore, an enrichment in mRNA processing proteins was observed in two previous HIV pull-down assays that used HIV$15^{\prime}$ leader sequence and unspliced RNA (Vallejos et al., 2011; Knoener et al., 2017). Although the pull-down methods and cell types used in both these studies were distinct, common proteins with our tat RNA pull-down assay were identified such as HNRNPL, HNRNPU, SRP14, and TERA that were isolated from HeLa cells arrested at G2/M with the HIV1 5'UTRgag (Vallejos et al., 2011), as well as PPIA, GSTP1, STIP1, PHB, NUDC, FLNB, FUBP1, DEK, MAP4, CLIC1, and CD47 (Supplementary Table 4) identified from Jurkat cells infected with NL4-3 and unspliced HIV-1 RNA-cellular protein complexes (Knoener et al., 2017). 

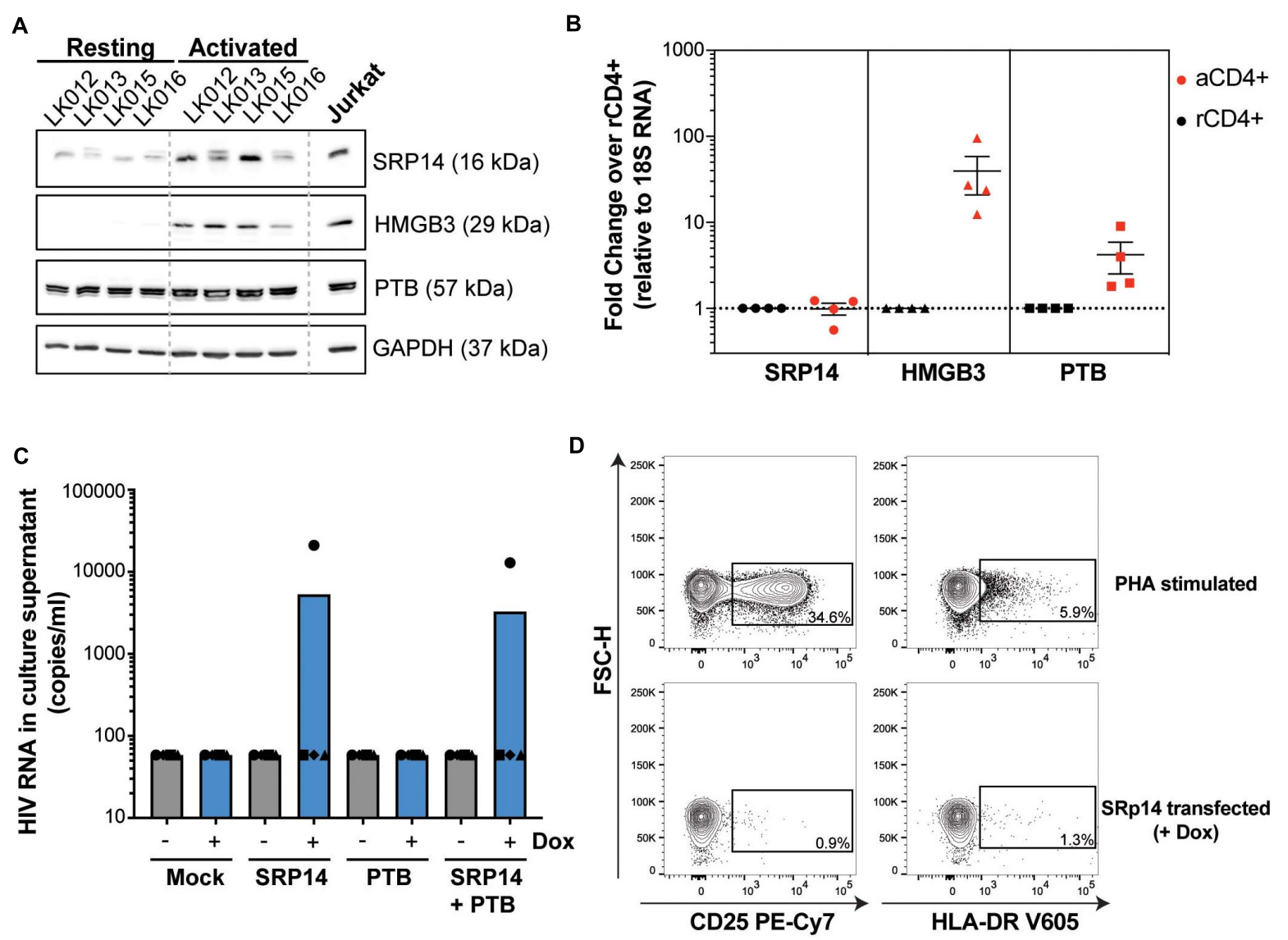

FIGURE 7 | SRP14 and PTB expression induce HIV expression in primary CD4+ T-cells from patients on ART. (A) SRP14, HMGB3 and PTB protein expression in resting $\mathrm{CD}_{4}{ }^{+} \mathrm{T}$-cells isolated from patient under antiretroviral therapy (LK, leukophoresis) increase in response to T-cell stimulation by $\alpha-\mathrm{CD} 3 / \mathrm{CD} 28(1 \mu \mathrm{g} / \mathrm{mL} ; 0.5$ $\mu \mathrm{g} / \mathrm{mL}$ ). Expression of SRP14, HMGB3, and PTB was measured by western-blot in resting and activated CD4 + T-cells $72 \mathrm{~h}$ post-stimulation. GAPDH was used as a loading control and Jurkat protein lysate as a positive control for antibody detection of the various proteins. (B) SRP14, HMGB3, and PTB RNA levels were assessed by RT-qPCR and shown as fold change in activated $\left(\mathrm{aCD} 4^{+}\right)$vs. resting $\mathrm{CD} 4^{+}\left(\mathrm{rCD}^{+}\right)$after normalization on 18S RNA. (C) Effect of SRP14, HMGB3 and PTB overexpression following doxycycline (+Dox) treatment on HIV-1 RNA levels in the culture supernatant $48 \mathrm{~h}$ post-stimulation. (D) Plots depicting the expression of middle (CD25) and late (HLA-DR) activation markers on SRP14 transfected cells and PHA stimulated cells.

It should be noted that the large isoform of PTB, PTBP1, can overcome the nuclear retention of MS RNA in latently infected cells when overexpressed (Lassen et al., 2006). However, the levels detected in resting $\mathrm{CD} 4^{+} \mathrm{T}$-cells from patients on ART were no different to activated $\mathrm{CD} 4^{+}$T-cells. Hence the specific isoform involved in Tat expression may be distinct to the isoform responsible for reversing aberrant accumulation of MS RNA. Further studies are required to determine the contribution of PTB to latency.

SRP14 (Signal Recognition Particle 14) is part of the signal recognition particle RNP complex, which functions by arresting the ribosome during translation so that secretory proteins can be correctly targeted to the endoplasmic reticulum (Weichenrieder et al., 2000; Lakkaraju et al., 2008). SRP14 together with SRP9 inhibits both cap- and IRES-dependent initiation. By binding to $40 \mathrm{~S}$ ribosomal subunits, SRP9/14 prevents $48 \mathrm{~S}$ complex formation hence interfering with the recruitment of mRNA to 40 S subunits (Ivanova et al., 2015). HMGB3 (High Mobility Group Box 3) is thought to be a DNA-binding protein that can remodel chromatin structures and can also act as a nucleic acid sensor-these are putative functions inferred from its shared homology with other proteins in the HMG-Box family (Nemeth et al., 2003). An analysis of the entire mRNA bound proteome in HEK 293 cells, however, identified HMGB3 as a potential RNAbinding protein (Baltz et al., 2012) although to date, no specific RNA binding associated function has been reported for HMGB3. In the present study, SRP14 and HMGB3 appear to have a positive and inhibitory role on Tat IRES translation, respectively. To our knowledge, these findings are the first to link SRP14 and HMGB3 to HIV expression. The exact mechanisms of which require investigation.

Our findings highlight SRP14 and HMGB3 as potential targets of pharmaceutical intervention. This still does not present an ideal situation as, akin to LRAs used in the past, targeting of these cellular proteins do not confer specificity against latent HIV-1. These proteins, however, are unlikely to have an involvement in gene expression pathways as extensive as that of the epigenetic modifiers and proteins involved in the NFKB and 


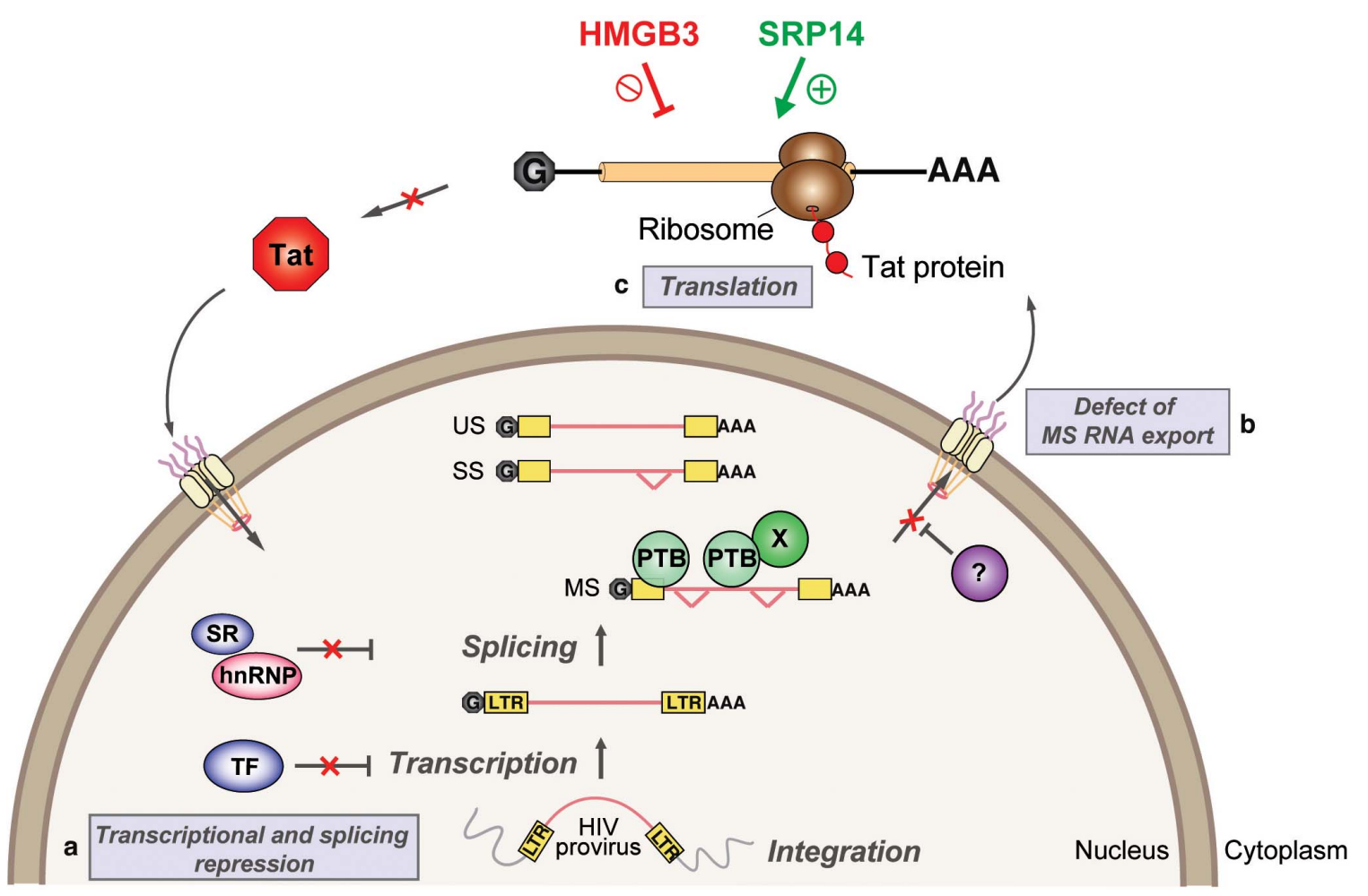

FIGURE 8 | Model depicting SRP14 and HMGB3 roles in post-transcriptional regulation of HIV-1 gene expression in latently infected cells. Multiple factors have been implicated in the establishment and maintenance of latency including (a) transcriptional and splicing repression by sequestration of essential transcription factors (TF) and/or splicing factors; (b) defect in multiply spliced (MS) RNA export due to insufficient levels of Rev and/or RNA binding factors such as PTB (for polypyrimidine tract binding protein); and (c) aberrant localization of MS RNA in the nucleus imposes a block on translation. Due to its central role in transcriptional transactivation and production of full-length viral mRNAs, small stochastic changes in Tat expression, the absence or impairment of Tat function would favor latent infection. By targeting tat mRNA, SRP14 activates Tat translation and hence stimulates HIV replication, while HMGB3 protein inhibits Tat translation and consequently impedes HIV expression in resting CD4 ${ }^{+} \mathrm{T}$ cells.

NFAT pathways, and hence may only impact upon the expression of a limited array of cellular genes.

There are several limitations in this study that should be acknowledged. Given the large number of cells required to complete the pull-down assay, we chose J-Lat cells to use for the screening of Tat RNA binding proteins during latency given their transformed nature and homogeneity of the cell population. While latently infected T-cell lines are useful for screening, a common limitation of using T-cell lines is that the intrinsic cellular factors and environment in T-cell lines are very different to that of primary T-lymphocytes hence the cellular factors that influence HIV latency may differ between T-cell lines and primary $\mathrm{CD}^{+}$T-cells. Another caveat is the pulldown assay was performed on a mixed population of productively infected cells, as $100 \%$ reactivation of J-Lat cells is never achieved even after treatment with strong mitogens, including TNF- $\alpha$. Virus expression from HIV latently infected T-cells could be sorted into two sub-populations; inducible $\left(\mathrm{GFP}^{+}\right)$and noninducible proviruses (GFP-), following treatment with TNF$\alpha$. The reporter systems used in this study assess different stages of the Tat expression pathway. However, these assays do not allow evaluation of these stages independently of the others. Further validation studies are required to determine the exact mechanisms exerted by SRP14 and HMGB3 on the pathways of Tat expression. Finally, in this study we did not address whether SRP14 expression is sufficient to circumvent MS RNA retention in the nucleus of resting $\mathrm{CD}^{+}{ }^{+}$cells, and whether it can induce production of replication competent virus. Further experiments are also required to test the effect of HMGB3 and SRP14 on tat mRNA export and/or stability.

Reactivation of HIV-1 can result from fluctuations in the levels of Tat protein (Weinberger et al., 2005; Khoury et al., 2020) as well as cellular factors involved in reinforcement of silent infection (Burnett et al., 2009). Hence, targeting Tat expression may serve as the basis for development of a more biologically relevant "shock and kill" strategy, a process that could lead to the discovery of an effective and durable functional cure for HIV-1.

\section{DATA AVAILABILITY STATEMENT}

The datasets presented in this study can be found in online repositories. The names of the repository/repositories and accession number(s) can be found below: PRIDE, PXD025782. 


\section{ETHICS STATEMENT}

The studies involving the use of blood samples from HIV negative donors were reviewed and approved by the Human Research and Ethics Committees from the University of Melbourne (1509VIC-03 and 17-08VIC-01). All HIV-1 seronegative donors were recruited by the Red Cross Blood Bank (Melbourne, Australia) and provided written informed consent for the use of their blood products for the research. The use of blood samples from people living with HIV was approved by the Alfred Hospital (HREC214/15) for the study entitled Large volume peripheral blood mononuclear cells (PBMCs) collection by leukapheresis to define HIV persistence in HIV-infected adults. All participants provided informed consent and the protocol was approved by the local Institutional Review Board. The patients/participants provided their written informed consent to participate in this study.

\section{AUTHOR CONTRIBUTIONS}

GK, ML, and DP conceived and designed the study. GK, ML, and SR acquired the data. GK, ML, SS, and DP analyzed and interpreted the data. JM, AP, and SL provided resources. GK and ML drafted the manuscript. All authors read and approved the final version of the manuscript.

\section{FUNDING}

This study was supported by the National Health and Medical Research Council of Australia (NHMRC) program and project grants (\#1052979 and \#1129320 to DP), NHMRC Principal Research Fellowship (\#1137739 to AP) and Australian Centre for HIV and Hepatitis Virology Research $\left(\mathrm{ACH}^{2}\right)$ (2015-21 to DP).

\section{ACKNOWLEDGMENTS}

We would like to thank the Department of Infectious Diseases at the Alfred Hospital and the Lewin/Cameron Clinical Research Group at the Peter Doherty Institute for providing the leukapheresis samples. We thank L. Wakim for providing the pInducer10 lentivector and her insight into T-cell infections. We thank M. Olshansky for his assistance in the bioinformatics analysis, N. Peirce, D. Yurick, J. Edwards, and A. Uldrich for their technical help. We thank the DMI Flow Facility staff for their advice and generous assistance in maintaining the flow cytometers and sorters used in this study. We also acknowledge

\section{REFERENCES}

Ait-Ammar, A., Kula, A., Darcis, G., Verdikt, R., De Wit, S., Gautier, V., et al. (2020). Current status of latency reversing agents facing the heterogeneity of HIV-1 cellular and tissue reservoirs. Front. Microbiol. 10:3060. doi: 10.3389/ fmicb.2019.03060 instrumentation, training and technical support by the Monash Biomedical Proteomics Facility. Computational resources were supported by the R@CMon/Monash Node of the Nectar Research Cloud, an initiative of the Australian Government's Super Science Scheme and the Education Investment Fund.

\section{SUPPLEMENTARY MATERIAL}

The Supplementary Material for this article can be found online at: https://www.frontiersin.org/articles/10.3389/fgene. 2021.680725/full\#supplementary-material

Supplementary Figure 1 | GO Biological Process analysis of the full set of proteins detected by mass spectrometry. List of proteins that were uniquely detected in the pull-down assay using untreated J-Lat 6.3 (A) or TNF- $\alpha$ activated (B) lysates were analyzed through Enrichr and overrepresented GO terms are depicted in the bar graphs. Examples of proteins belonging to the top classes are listed.

Supplementary Figure 2 | Protein and mRNA profiles of KD RFP + Jurkat cells. (A) Representative histograms of RFP ${ }^{+}$shRNA transduced Jurkats (bulk cells) where SRP14, HMGB3 or PTB were targeted for knockdown. (B) Representative western blots showing knockdown in protein levels of the three genes of interest, SRP14, HMGB3, PTB in their respective bulk shRNA-generated cell lines. Numbers below the blots show relative amounts of proteins in comparison to untransduced Jurkats. (C) Expression of RFP in bulk or single clone Jurkat cells targeted for knockdown of SRP14, HMGB3 or PTB. (D) Fold change in SRP14, HMGB3 and PTB mRNA levels in their respective bulk shRNA-generated cell lines or single clones compared to untreated Jurkat cells as determined by RT-qPCR.

Supplementary Figure 3 | R7GEmTB reporter virus label latently infected cells. Jurkat and $\mathrm{CD}^{+}{ }^{+}$T-cells were infected with R7GEmTB dual color reporter virus and subjected to confocal microscopy to assess the infection phenotype. $\mathrm{GFP}^{+}$and $\mathrm{GFP}^{+} \mathrm{BFP}^{+}$cells, representative of productive infection are shown in green and cyan, while $\mathrm{BFP}^{+}$cells representative of latent infection are shown in blue.

Supplementary Figure 4 | Western-blot analysis of RNP complexes. Analysis of the protein content of the RNP complexes formed on tat mRNA by western-blot using antibodies directed against SRP14 and HMGB3. MBP-MS2 was used as a loading control (ponceau stain). JL- and $\mathrm{JL}+$ represent total lysates prepared from J-Lat 6.3 left untreated or treated with TNF- $\alpha$, respectively.

Supplementary Table 1 | Participant demographics.

Supplementary Table 2 | Primers and probes used in this study. The number, sequence, and usage of each primer are given. Restriction sites are underlined, start and stop codons are in boldfaces.

Supplementary Table 3 | List of protein hits selected for follow-up analysis. Summary of the gene ID, uniprot, gene symbol, protein name, function, mass (in $\mathrm{kDa}$ ), and protein length (in amino acid, aa) are indicated.

Supplementary Table 4 | List of proteins identified by affinity purification coupled to mass spectrometry in the RNP complexes formed with 5'UTRtat1-Tat-MS2 and Tat-MS2 RNAs. The number of peptides identified for each bait (5'UTRtat-Tat-MS2 and Tat-MS2) and prey (protein) as well as their fold-enrichment in resting and activated cells are indicated. 
Baltz, A. G., Munschauer, M., Schwanhausser, B., Vasile, A., Murakawa, Y., Schueler, M., et al. (2012). The mRNA-bound proteome and its global occupancy profile on protein-coding transcripts. Mol. Cell. 46, 674-690. doi: 10.1016/j.molcel.2012.05.021

Bar, A., Marchand, V., Khoury, G., Dreumont, N., Mougin, A., Robas, N., et al. (2011). Structural and functional analysis of the Rous Sarcoma virus negative regulator of splicing and demonstration of its activation by the 9G8 SR protein. Nucleic Acids Res. 39, 3388-3403. doi: 10.1093/nar/gkq1114

Brass, A. L., Dykxhoorn, D. M., Benita, Y., Yan, N., Engelman, A., Xavier, R. J., et al. (2008). Identification of host proteins required for HIV infection through a functional genomic screen. Science 319, 921-926. doi: 10.1126/science.1152725

Burnett, J. C., Miller-Jensen, K., Shah, P. S., Arkin, A. P., and Schaffer, D. V. (2009). Control of stochastic gene expression by host factors at the HIV promoter. PLoS Pathog. 5:e1000260. doi: 10.1371/journal.ppat.1000260

Calvanese, V., Chavez, L., Laurent, T., Ding, S., and Verdin, E. (2013). Dual-color HIV reporters trace a population of latently infected cells and enable their purification. Virology 446, 283-292. doi: 10.1016/j.virol.2013.07.037

Charnay, N., Ivanyi-Nagy, R., Soto-Rifo, R., Ohlmann, T., Lopez-Lastra, M., and Darlix, J. L. (2009). Mechanism of HIV-1 Tat RNA translation and its activation by the Tat protein. Retrovirology 6:74. doi: 10.1186/1742-4690-6-74

Chiu, Y. L., Coronel, E., Ho, C. K., Shuman, S., and Rana, T. M. (2001). HIV-1 Tat protein interacts with mammalian capping enzyme and stimulates capping of TAR RNA. J. Biol. Chem. 276, 12959-12966. doi: 10.1074/jbc.m007901200

Chiu, Y.-L., Ho, C. K., Saha, N., Schwer, B., Shuman, S., and Rana, T. M. (2002). Tat stimulates cotranscriptional capping of HIV mRNA. Mol. Cell. 10, 585-597. doi: 10.1016/s1097-2765(02)00630-5

Clouse, K. A., Powell, D., Washington, I., Poli, G., Strebel, K., Farrar, W., et al. (1989). Monokine regulation of human immunodeficiency virus-1 expression in a chronically infected human T cell clone. J. Immunol. 142, 431-438.

Cvitkovic, I., and Jurica, M. S. (2013). Spliceosome database: a tool for tracking components of the spliceosome. Nucleic Acids Res. 41, 132-141.

Deckert, J., Hartmuth, K., Boehringer, D., Behzadnia, N., Will, C. L., Kastner, B., et al. (2006). Protein composition and electron microscopy structure of affinity-purified human spliceosomal B complexes isolated under physiological conditions. Mol. Cell Biol. 26, 5528-5543. doi: 10.1128/mcb.00582-06

Deeks, S. G., Lewin, S. R., Ross, A. L., Ananworanich, J., Benkirane, M., Cannon, P., et al. (2016). International AIDS Society global scientific strategy: Towards an HIV cure 2016. Nat. Med. 22, 839-850.

Donahue, D. A., Kuhl, B. D., Sloan, R. D., and Wainberg, M. A. (2012). The viral protein Tat can inhibit the establishment of HIV-1 latency. J. Virol. 86, 3253-3263. doi: 10.1128/jvi.06648-11

Edgcomb, S. P., Carmel, A. B., Naji, S., Ambrus-Aikelin, G., Reyes, J. R., Saphire, A. C. S., et al. (2012). DDX1 is an RNA-dependent ATPase involved in HIV-1 Rev function and virus replication. J. Mol. Biol. 415, 61-74. doi: 10.1016/j.jmb. 2011.10.032

Folks, T. M., Clouse, K. A., Justement, J., Rabson, A., Duh, E., Kehrl, J. H., et al. (1989). Tumor necrosis factor alpha induces expression of human immunodeficiency virus in a chronically infected T-cell clone. Proc. Natl. Acad. Sci. U S A 86, 2365-2368. doi: 10.1073/pnas.86.7.2365

Gallo, D. E., and Hope, T. J. (2012). Knockdown of MAP4 and DNAL1 produces a post-fusion and pre-nuclear translocation impairment in HIV-1 replication. Virology. 422, 13-21. doi: 10.1016/j.virol.2011.09.015

Ivanova, E., Berger, A., Scherrer, A., Alkalaeva, E., and Strub, K. (2015). Alu RNA regulates the cellular pool of active ribosomes by targeted delivery of SRP9/14 to 40 S subunits. Nucleic Acids Res. 43, 2874-2887. doi: 10.1093/nar/gkv048

Jablonski, J. A., Amelio, A. L., Giacca, M., and Caputi, M. (2010). The transcriptional transactivator Tat selectively regulates viral splicing. Nucleic Acids Res. 38, 1249-1260. doi: 10.1093/nar/gkp1105

Jäger, S., Cimermancic, P., Gulbahce, N., Johnson, J. R., McGovern, K. E., Clarke, S. C., et al. (2011). Global landscape of HIV-human protein complexes. Nature $481,365-370$.

Jordan, A., Bisgrove, D., and Verdin, E. (2003). HIV reproducibly establishes a latent infection after acute infection of T cells in vitro. EMBO J. 22, 1868-1877. doi: 10.1093/emboj/cdg188

Karabiber, F., McGinnis, J. L., Favorov, O. V., and Weeks, K. M. (2013). QuShape: rapid, accurate, and best-practices quantification of nucleic acid probing information, resolved by capillary electrophoresis. RNA 19, 63-73. doi: 10 . 1261/rna.036327.112
Khoury, G., Darcis, G., Lee, M. Y., Bouchat, S., Van Driessche, B., Purcell, D. F., et al. (2018a). The Molecular Biology of HIV Latency. In: HIV vaccine and cure - The Path Towards Finding an Effective Cure and Vaccine. Cham: Springer Nature.

Khoury, G., Mota, T. M., Li, S., Tumpach, C., Lee, M. Y., Jacobson, J., et al. (2018b). HIV latency reversing agents act through Tat post translational modifications. Retrovirology 15:36.

Khoury, G., Mackenzie, C., Ayadi, L., Lewin, S. R., Branlant, C., and Purcell, D. F. J. (2020). Tat IRES modulator of tat mRNA (TIM-TAM): a conserved RNA structure that controls Tat expression and acts as a switch for HIV productive and latent infection. Nucleic Acids Res. 48, 2643-2660. doi: 10.1093/ nar/gkz1181

Knoener, R. A., Becker, J. T., Scalf, M., Sherer, N. M., and Smith, L. M. (2017). Elucidating the in vivo interactome of HIV-1 RNA by hybridization capture and mass spectrometry. Sci. Rep. 7:16965.

König, R., Zhou, Y., Elleder, D., Diamond, T. L., Bonamy, G. M. C., Irelan, J. T., et al. (2008). Global analysis of host-pathogen interactions that regulate early-stage HIV-1 Replication. Cell 135, 49-60. doi: 10.1016/j.cell.2008.07.032

Krueger, B. J., Varzavand, K., Cooper, J. J., and Price, D. H. (2010). The mechanism of release of P-TEFb and HEXIM1 from the 7SK snRNP by viral and cellular activators includes a conformational change in 7SK. PLoS One. 5:e12335. doi: 10.1371/journal.pone.0012335

Lakkaraju, A. K., Mary, C., Scherrer, A., Johnson, A. E., and Strub, K. (2008). SRP keeps polypeptides translocation-competent by slowing translation to match limiting ER-targeting sites. Cell 133, 440-451. doi: 10.1016/j.cell.2008.02. 049

Lassen, K. G., Ramyar, K. X., Bailey, J. R., Zhou, Y., and Siliciano, R. F. (2006). Nuclear retention of multiply spliced HIV-1 RNA in resting CD4+ T cells. PLoS Pathog. 2:e68. doi: 10.1371/journal.ppat.0020068

Liu, J., Henao-Mejia, J., Liu, H., Zhao, Y., and He, J. J. (2011). Translational regulation of HIV-1 replication by HIV-1 rev cellular cofactors Sam68, eIF5A, hRIP, and DDX3. J. Neuroimmun. Pharmacol. 6, 308-321. doi: 10.1007/s11481011-9265-8

Lozano, G., and Martínez-Salas, E. (2015). Structural insights into viral IRESdependent translation mechanisms. Curr. Opin. Virol. 12, 113-120. doi: 10. 1016/j.coviro.2015.04.008

Maenner, S., Blaud, M., Fouillen, L., Savoye, A., Marchand, V., Dubois, A., et al. (2010). 2-D structure of the A region of Xist RNA and its implication for PRC2 association. PLoS Biol. 8:e1000276. doi: 10.1371/journal.pbio.1000276

Meerbrey, K. L., Hu, G., Kessler, J. D., Roarty, K., Li, M. Z., Fang, J. E., et al. (2011). The pINDUCER lentiviral toolkit for inducible RNA interference in vitro and in vivo. Proc. Natl. Acad. Sci. U S A. 108, 3665-3670. doi: 10.1073/pnas. 1019736108

Monette, A., Ajamian, L., Lopez-Lastra, M., and Mouland, A. J. (2009). Human immunodeficiency virus type 1 (HIV-1) induces the cytoplasmic retention of heterogeneous nuclear ribonucleoprotein A1 by disrupting nuclear import: implications for HIV-1 gene expression. J. Biol. Chem. 284, 31350-31362.

Moron-Lopez, S., Telwatte, S., Sarabia, I., Battivelli, E., Montano, M., Macedo, A. B., et al. (2020). Human splice factors contribute to latent HIV infection in primary cell models and blood CD4+ T cells from ART-treated individuals. PLoS Pathog. 16:e1009060. doi: 10.1371/journal.ppat.1009060

Mortimer, S. A., and Weeks, K. M. (2007). A fast-acting reagent for accurate analysis of RNA secondary and tertiary structure by SHAPE chemistry. J. Am. Chem. Soc. 129, 4144-4145. doi: 10.1021/ja0704028

Muniz, L., Egloff, S., Ughy, B., Jády, B. E., and Kiss, T. (2010). Controlling cellular P-TEFb activity by the HIV-1 transcriptional transactivator tat. PLoS Pathog. 6:e1001152. doi: 10.1371/journal.ppat.1001152

Nemeth, M. J., Curtis, D. J., Kirby, M. R., Garrett-Beal, L. J., Seidel, N. E., Cline, A. P., et al. (2003). Hmgb3: an HMG-box family member expressed in primitive hematopoietic cells that inhibits myeloid and B-cell differentiation. Blood 102, 1298-1306. doi: 10.1182/blood-2002-11-3541

Nguyen, W., Jacobson, J., Jarman, K. E., Jousset Sabroux, H., Harty, L., McMahon, J., et al. (2019). Identification of 5-substituted 2-acylaminothiazoles that activate Tat mediated transcription in HIV-1 latency models. J. Med. Chem. 62, 51485175. doi: 10.1021/acs.jmedchem.9b00462

Ott, M., Geyer, M., and Zhou, Q. (2011). The control of HIV transcription: keeping RNA polymerase II on track. Cell Host. Microb. 10, 426-435. doi: 10.1016/j. chom.2011.11.002 
Pasternak, A. O., and Berkhout, B. (2018). What do we measure when we measure cell-associated HIV RNA. Retrovirology 15:13.

Perez-Riverol, Y., Csordas, A., Bai, J., Bernal-Llinares, M., Hewapathirana, S., Kundu, D. J., et al. (2019). The PRIDE database and related tools and resources in 2019: improving support for quantification data. Nucleic Acids Res. 47, D442-D450.

Ping, Y.-H., and Rana, T. M. (2001). DSIF and NELF Interact with RNA Polymerase II Elongation Complex and HIV-1 Tat Stimulates P-TEFb-mediated Phosphorylation of RNA Polymerase II and DSIF during Transcription Elongation *. J. Biol. Chem. 276, 12951-12958. doi: 10.1074/jbc.m006130200

Razooky, B. S., Pai, A., Aull, K., Rouzine, I. M., and Weinberger, L. S. (2015). A hardwired HIV latency program. Cell 160, 990-1001. doi: 10.1016/j.cell.2015. 02.009

Rivas-Aravena, A., Ramdohr, P., Vallejos, M., Valiente-Echeverría, F., DormoyRaclet, V., Rodríguez, F., et al. (2009). The Elav-like protein HuR exerts translational control of viral internal ribosome entry sites. Virology. 392, 178185. doi: 10.1016/j.virol.2009.06.050

Ropers, D., Ayadi, L., Gattoni, R., Jacquenet, S., Damier, L., Branlant, C., et al. (2004). Differential effects of the SR proteins 9G8, SC35, ASF/SF2, and SRp40 on the utilization of the A1 to A5 splicing sites of HIV-1 RNA. J. Biol. Chem. 279, 29963-29973. doi: 10.1074/jbc.m404452200

Saleh, S., Wightman, F., Ramanayake, S., Alexander, M., Kumar, N., Khoury, G., et al. (2011). Expression and reactivation of HIV in a chemokine induced model of HIV latency in primary resting CD4+ T cells. Retrovirology 8:80. doi: $10.1186 / 1742-4690-8-80$

Saliou, J. M., Bourgeois, C. F., Ayadi-Ben Mena, L., Ropers, D., Jacquenet, S., Marchand, V., et al. (2009). Role of RNA structure and protein factors in the control of HIV-1 splicing. Front. Biosci. 14, 2714-2729. doi: 10.2741/ 3408

Sarzotti-Kelsoe, M., Bailer, R. T., Turk, E., Lin, C. L., Bilska, M., Greene, K. M., et al. (2014). Optimization and validation of the TZM-bl assay for standardized assessments of neutralizing antibodies against HIV-1. J. Immunol. Methods. 409, 131-146. doi: 10.1016/j.jim.2013.11.022

Schapira, M., Raaka, B. M., Das, S., Fan, L., Totrov, M., Zhou, Z., et al. (2003). Discovery of diverse thyroid hormone receptor antagonists by high-throughput docking. Proc. Natl. Acad. Sci. U S A. 100, 7354-7359. doi: 10.1073/pnas. 1131854100

Spina, C. A., Anderson, J., Archin, N. M., Bosque, A., Chan, J., Famiglietti, M., et al. (2013). An in-depth comparison of latent HIV-1 reactivation in multiple cell model systems and resting CD4+ T cells from aviremic patients. PLoS Pathog. 9:e1003834. doi: 10.1371/journal.ppat.1003834
Vallejos, M., Deforges, J., Plank, T. D., Letelier, A., Ramdohr, P., Abraham, C. G., et al. (2011). Activity of the human immunodeficiency virus type 1 cell cycledependent internal ribosomal entry site is modulated by IRES trans-acting factors. Nucleic Acids Res. 39, 6186-6200. doi: 10.1093/nar/gkr189

Weichenrieder, O., Wild, K., Strub, K., and Cusack, S. (2000). Structure and assembly of the Alu domain of the mammalian signal recognition particle. Nature 408, 167-173. doi: 10.1038/3504 1507

Weinberger, L. S., Burnett, J. C., Toettcher, J. E., Arkin, A. P., and Schaffer, D. V. (2005). Stochastic gene expression in a lentiviral positive-feedback loop: HIV-1 Tat fluctuations drive phenotypic diversity. Cell 122, 169-182. doi: 10.1016/j. cell.2005.06.006

Yukl, S., Pillai, S., Li, P., Chang, K., Pasutti, W., Ahlgren, C., et al. (2009). Latentlyinfected CD4+ T cells are enriched for HIV-1 Tat variants with impaired transactivation activity. Virology 387, 98-108. doi: 10.1016/j.virol.2009.01.013

Yukl, S. A., Kaiser, P., Kim, P., Telwatte, S., Joshi, S. K., Vu, M., et al. (2018). HIV latency in isolated patient $\mathrm{CD} 4+\mathrm{T}$ cells may be due to blocks in HIV transcriptional elongation, completion, and splicing. Sci. Transl. Med. 10:eaa9927.

Zerbato, J. M., Khoury, G., Zhao, W., Gartner, M. J., Pascoe, R. D., Rhodes, A., et al. (2021). Multiply spliced HIV RNA is a predictive measure of virus production ex vivo and in vivo following reversal of HIV latency. EBioMedicine 65:103241. doi: 10.1016/j.ebiom.2021.103241

Zerbato, J. M., Purves, H. V., Lewin, S. R., and Rasmussen, T. A. (2019). Between a shock and a hard place: challenges and developments in HIV latency reversal. Curr. Opin. Virol. 38, 1-9. doi: 10.1016/j.coviro.2019.03.004

Zhou, H., Xu, M., Huang, Q., Gates, A. T., Zhang, X. D., Castle, J. C., et al. (2008). Genome-scale RNAi screen for host factors required for HIV replication. Cell. Host. Microb. 4, 495-504. doi: 10.1016/j.chom.2008.10.004

Conflict of Interest: The authors declare that the research was conducted in the absence of any commercial or financial relationships that could be construed as a potential conflict of interest.

Copyright (C) 2021 Khoury, Lee, Ramarathinam, McMahon, Purcell, Sonza, Lewin and Purcell. This is an open-access article distributed under the terms of the Creative Commons Attribution License (CC BY). The use, distribution or reproduction in other forums is permitted, provided the original author(s) and the copyright owner(s) are credited and that the original publication in this journal is cited, in accordance with accepted academic practice. No use, distribution or reproduction is permitted which does not comply with these terms. 